\title{
Penrose Process: Its Variants and Astrophysical Applications
}

\author{
Zdeněk Stuchlík*(D), Martin Kološ (D) and Arman Tursunov (D) \\ Research Centre for Theoretical Physics and Astrophysics, Institute of Physics, Silesian University in Opava, \\ Bezručovo nám.13, CZ-74601 Opava, Czech Republic; martin.kolos@fpf.slu.cz (M.K.); \\ arman.tursunov@fpf.slu.cz (A.T.) \\ * Correspondence: zdenek.stuchlik@fpf.slu.cz
}

Citation: Stuchlík, Z.; Kološ, M.; Tursunov, A. Penrose Process: Its Variants and Astrophysical Applications. Universe 2021, 7, 416. https://doi.org/10.3390/ universe7110416

Academic Editors: Lorenzo Iorio and Ashkbiz Danehkar

Received: 23 September 2021

Accepted: 27 October 2021

Published: 31 October 2021

Publisher's Note: MDPI stays neutral with regard to jurisdictional claims in published maps and institutional affiliations.

Copyright: (c) 2020 by the authors. Licensee MDPI, Basel, Switzerland. This article is an open access article distributed under the terms and conditions of the Creative Commons Attribution (CC BY) license (https:/ / creativecommons.org/licenses/by/ $4.0 /)$.

\begin{abstract}
We present a review of the Penrose process and its modifications in relation to the Kerr black holes and naked singularities (superspinars). We introduce the standard variant of this process, its magnetic version connected with magnetized Kerr black holes or naked singularities, the electric variant related to electrically charged Schwarzschild black holes, and the radiative Penrose process connected with charged particles radiating in the ergosphere of magnetized Kerr black holes or naked singularities. We discuss the astrophysical implications of the variants of the Penrose process, concentrating attention to the extreme regime of the magnetic Penrose process leading to extremely large acceleration of charged particles up to ultra-high energy $E \sim 10^{22} \mathrm{eV}$ around magnetized supermassive black holes with mass $M \sim 10^{10} M_{\odot}$ and magnetic intensity $B \sim 10^{4} \mathrm{G}$. Similarly high energies can be obtained by the electric Penrose process. The extraordinary case is represented by the radiative Penrose process that can occur only around magnetized Kerr spacetimes but just inside their ergosphere, in contrast to the magnetic Penrose process that can occur in a more extended effective ergosphere determined by the intensity of the electromagnetic interaction. The explanation is simple, as the radiative Penrose process is closely related to radiated photons with negative energy whose existence is limited just to the ergosphere.
\end{abstract}

Keywords: rotating black holes; accretion disks; magnetic fields; ultra-high energy particles

\section{Introduction}

The Penrose process [1] introduces the extraction of rotational (or electrostatic) energy from rotating (charged) black holes, or their counterparts represented by naked singularities (or superspinars), by using the decay of particles in the ergosphere, a region of extremely strong influence of the spacetime rotation, allowing for the existence of particles with negative energy relative to distant observers. The present article is devoted to the Penrose process and its variants related to magnetized or electrically charged black holes.

Active galactic nuclei (quasars) radiate extremely large energy due to accretion disks orbiting a central supermassive black hole with mass $M>10^{6} M_{\odot}$ and jets created near the disk edge close to the black hole horizon [2]. It is generally assumed that creation of the jets is related to the Blandford-Znajek process [3] that can be treated as a collective demonstration of the magnetic Penrose process [4]. In a series of works [5,6], we have shown that the most efficient demonstration of the magnetic Penrose process is related to the ionized Keplerian (or toroidal) accretion disks.

The theory of accretion disks is very complex [7], being based on two approaches. The geometrically thin, Keplerian, accretion disks are basically governed by the spacetime circular geodesics [8]. The geometrically thick, toroidal accretion disks are basically governed by the effective potential implied by the Euler equation describing the balance of the gravitational forces, inertial forces, and pressure gradients of orbiting perfect fluid [9]. The equilibrium tori are related to the closed equipotential surfaces of the effective potential, accretion tori are related to the self-crossing equipotential surfaces, and jets are related to the open equipotential surfaces. The ringed accretion disks describe complex toroidal structures [10-12]. The role of the cosmic 
repulsion on the disk structures around supermassive black holes is summarized in [13,14]; it is strongest near the static (or turnaround) radius [15,16], giving a natural limit on gravitationally bounded systems in the accelerated Universe [17]. In our review, we are concentrated on physical processes in the vicinity of the black hole event horizon, namely, the ergosphere or effective ergosphere [18]. For completeness, we also comment on the situation that occurs around the Kerr naked singularities [19-21] or related Kerr superspinars [22-25].

All variants of the fate of ionized Keplerian disks were discussed in $[14,26]$. The magnetic Penrose process is relevant in the chaotic regime of the motion of the ionized matter of the innermost parts of the disk [27], allowing for acceleration to ultra-high energy [4,28]. The structure of magnetic fields around black holes is still under intensive debate, but the simple assumption of the uniform magnetic field [29] can be considered as a sufficient and highly illustrative approximation for discussion of the magnetic Penrose process [14].

In our review, we first present the standard "electrically neutral" form of the Penrose process and discuss its acceptability in astrophysical processes both for Kerr black holes and naked singularities. Then, we consider magnetized Kerr black holes (or naked singularities) and discuss the two regimes of the magnetic Penrose process and its applicability to the creation of ultra-high energy protons observed in cosmic rays; note that in the magnetic Penrose process the back-reaction effect due to radiation of the charge particles moving in the external magnetic field plays an important role [30,31]. Then, we present a new version-electric Penrose process, related to slightly charged non-rotating Schwarzschild black holes, where only the electrostatic energy can be extracted in the effective ergosphere of the black hole, demonstrating that even such a case could be astrophysically very efficient. Finally, we introduce the notion of a radiative Penrose process as a fundamentally new version that can be related to the radiative self-reaction that occurs solely in the ergosphere; although it can be realized only around magnetized Kerr black holes, the effective ergosphere of the radiating particle is irrelevant in this case.

Hereafter in this article, we derive the leading equations in the system of geometric units, in which $G=1=c$, unless the constants are written explicitly or the units are specified. For estimations of electric charges and magnetic fields, we use the cgs system of units, in which the electrostatic unit of charge is measured in Franklin, so that $1 \mathrm{Fr} \equiv 1 \mathrm{esu}=1 \mathrm{~cm}^{3 / 2} \mathrm{~g}^{1 / 2} \mathrm{~s}^{-1}$, while the magnetic field strength is measured in Gauss, so that $1 \mathrm{G}=1 \mathrm{~cm}^{-1 / 2} \mathrm{~g}^{1 / 2} \mathrm{~s}^{-1}$. Conversion to the SI system of units can be made as follows: $1 \mathrm{C}=3 \times 10^{9} \mathrm{Fr}$ and $1 \mathrm{~T}=10^{4} \mathrm{G}$.

\section{Kerr Black Holes and the Penrose Process}

The energy of Kerr black holes (also Kerr naked singularities or Kerr superspinars [22]) has a part related to their rotation that can be extracted in realistic astrophysical processes [32]. The simplest example of processes extracting rotational energy is the Penrose process [1], which is discussed in the present section.

\subsection{Kerr Geometry and Ergosphere}

Kerr black holes and naked singularities are governed by the line element that in the Boyer-Lindquist coordinates $(t, r, \theta, \phi)$, and the geometric units $(G=1=c)$, can be expressed in the form

$$
\mathrm{d} s^{2}=g_{t t} \mathrm{~d} t^{2}+2 g_{t \phi} \mathrm{d} t \mathrm{~d} \phi+g_{\phi \phi} \mathrm{d} \phi^{2}+g_{r r} \mathrm{~d} r^{2}+g_{\theta \theta} \mathrm{d} \theta^{2}
$$


where

$$
\begin{aligned}
& g_{t t}=-\left(1-\frac{2 M r}{\Sigma}\right), \\
& g_{t \phi}=-\frac{2 a M r \sin ^{2} \theta}{\Sigma}, \\
& g_{\phi \phi}=\left(r^{2}+a^{2}+\frac{2 a^{2} M r}{\Sigma} \sin ^{2} \theta\right) \sin ^{2} \theta, \\
& g_{r r}=\frac{\Sigma}{\Delta} \\
& g_{\theta \theta}=\Sigma
\end{aligned}
$$

with

$$
\Delta=r^{2}-2 M r+a^{2}, \Sigma=r^{2}+a^{2} \cos ^{2} \theta ;
$$

$M$ is the gravitational mass of the black hole [33] giving the mass in the Newtonian limit, $a=J / M$ is its spin, and $J$ is its internal angular momentum [18]. Assuming $a>0$, the Kerr black holes correspond to $a<M$; extreme Kerr black holes correspond to $a=M$; and Kerr naked singularities correspond to $a>M$. The physical singularity has a ring character, being located at $r=0, \theta=\pi / 2$; the Kerr spacetimes are discussed in detail in [34]. The Kerr metric (1) is asymptotically flat-for the generalization to the non-zero cosmological constant, see $[15,16]$.

The Kerr black hole energy represented by the mass parameter squared $M^{2}$ can be separated into two parts, namely, the irreducible and the rotational part, and takes the form $[35,36]$

$$
M^{2}=M_{i r}^{2}+\frac{J^{2}}{M_{i r}^{2}} ;
$$

the irreducible part of the black hole energy cannot be removed, and it is related to the black hole event horizon surface $S$ by the relation $S=16 \pi M_{i r}^{2}$, while the second rotational part is governed by the internal angular momentum of the black hole $J=a M$. The rotational part of the energy can be extracted by astrophysical processes $[1,3,32,37,38]$, and we shall discuss possible variants of this extraction in the particle form. Note that there is an additional wave form of the energy extraction called super-radiation related to the scattering of properly tuned waves [37].

It is useful to put for simplicity $M=1$ introducing thus dimensionless spin $a$ and dimensionless coordinates $r, t$. Sometimes it could also be useful to have Cartesian-like coordinates that can be obtained by the transformation to the Kerr-Schild coordinate system

$$
x=\sqrt{r^{2}+a^{2}} \sin \theta \cos \tilde{\phi}, \quad y=\sqrt{r^{2}+a^{2}} \sin \theta \sin \tilde{\phi}, \quad z=r \cos \theta,
$$

with $\tilde{\phi}=\phi-\tan ^{-1}(a / r)$.

The divergence of the metric coefficient $g_{r r}$ determines the horizons of the Kerr black holes located at

$$
r_{ \pm}=1 \pm\left(1-a^{2}\right)^{1 / 2} .
$$

The outer event horizon $r_{+}=1+\left(1-a^{2}\right)^{1 / 2}$ represents a boundary of the black hole with respect to distant observers. The inner horizon is a Cauchy horizon of the external spacetime [34]. In the extreme Kerr black holes, these horizon coincide at $r=1$. No event horizon exists in the Kerr naked singularity spacetimes.

We restrict to the black hole spacetime region located above the event horizon at $r_{+}$. The static observers exist only in the regions where $g_{t t}<0$. Above the event horizon, the static observers cannot exist in the ergosphere limited from above by the surface of stationarity, or stationary limit radius

$$
r_{\text {stat }+}(\theta)=1+\left(1-a^{2} \cos ^{2} \theta\right)^{1 / 2} .
$$


Under the inner horizon, the ergosphere is limited by the surface

$$
r_{\text {stat }-}(\theta)=1-\left(1-a^{2} \cos ^{2} \theta\right)^{1 / 2} .
$$

The shape of the ergosphere is illustrated in Figure 1; notice that for black holes, the ergosphere extends to the rotation (symmetry) axis, but this is not the case for naked singularities.
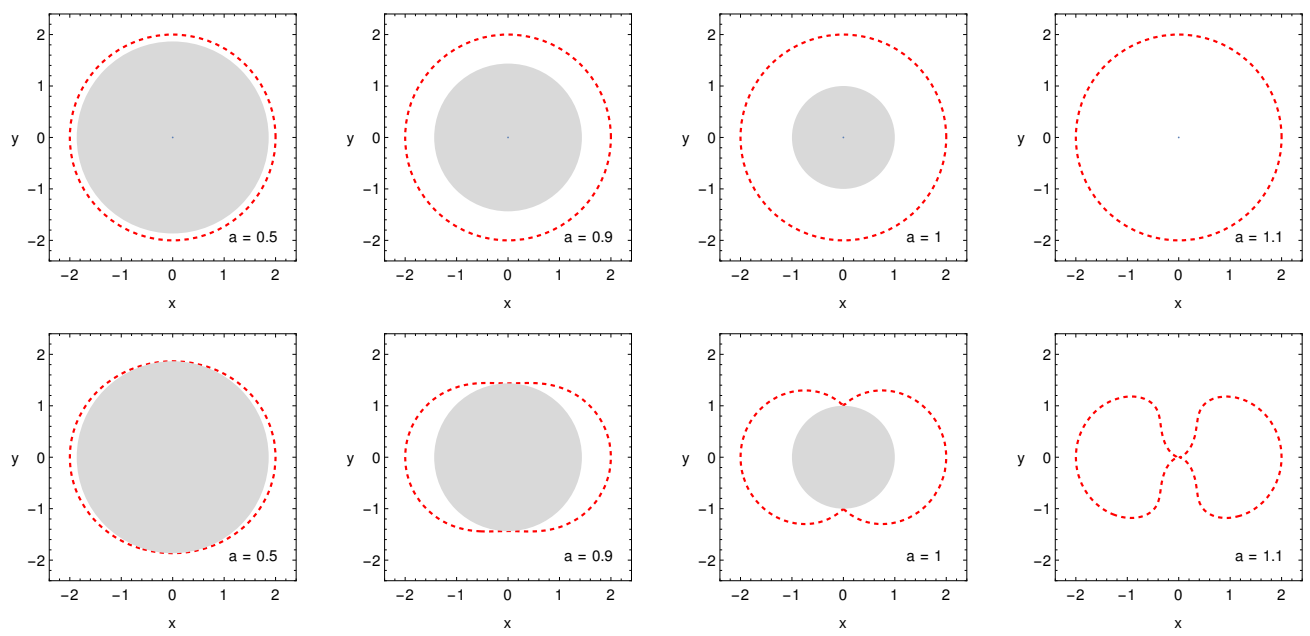

Figure 1. The ergosphere extension, represented by the equatorial and the meridional sections, is given for Kerr black holes and Kerr naked singularity.

\subsection{Test Particle Motion and Locally Non-Rotating Frames}

Motion of (uncharged) test particles having rest mass $m$ is governed by the geodesic equation

$$
\frac{D p^{\mu}}{D \tau}=0
$$

complemented by the normalization condition

$$
p^{\mu} p_{\mu}=\delta
$$

where $p^{\mu}$ is the particle four-momentum and $\delta=-m^{2}$ for massive particles, while $\delta=0$ for massless particles. Two Killing vector fields of the Kerr geometry, $\partial / \partial t$, and $\partial / \partial \phi$, imply the existence of conserved energy $E$ and axial angular momentum $L$.

As all the particles are dragged by the rotating spacetime, it is useful to determine limits on the angular velocity $\Omega=d \phi / d t$ of the orbiting matter (fixed at a given radius $r>r_{+}$) - the limits correspond to the motion of photon in the sense of rotation and in the opposite one. We thus find that the angular velocity of any circulating particle has to be limited by the interval

$$
\Omega_{+}>\Omega>\Omega_{-}
$$

where the restricting angular velocities are given by the relation

$$
\Omega_{ \pm}=-\frac{g_{t \phi}}{g_{\phi \phi}} \pm \sqrt{\left(\frac{g_{t \phi}}{g_{\phi \phi}}\right)^{2}-\frac{g_{t t}}{g_{\phi \phi}}} .
$$


Now we can directly see that we can define in the Kerr geometry the notion of the locally non-rotating frames (LNRF), related to the zero angular momentum observers (ZAMO) with axial angular momentum $L=0$, and four-velocity

$$
\begin{array}{r}
u_{\mathrm{LNRF}}^{\alpha}=\left(u_{\mathrm{LNRF}}^{t}, 0,0, u_{\mathrm{LNRF}}^{\phi}\right), \quad\left(u_{\mathrm{LNRF}}^{t}\right)^{2}=\frac{g_{\phi \phi}}{g_{t \phi}^{2}-g_{t t} g_{\phi \phi}}, \quad u_{\mathrm{LNRF}}^{\phi}=\Omega_{\mathrm{LNRF}} u_{\mathrm{LNRF}}^{t}, \\
\Omega_{\mathrm{LNRF}}(r, \theta)=-\frac{g_{t \phi}}{g_{\phi \phi}}=\frac{2 a r}{\left(r^{2}+a^{2}\right)^{2}-a^{2} \Delta \sin ^{2} \theta^{2}} .
\end{array}
$$

The LNRFs (ZAMOs) four-velocity are well defined above the horizon $\left(r>r_{+}\right)$in the black hole case and for all radii in the naked singularity case and are corotating with the Kerr spacetime at fixed coordinates $r$ and $\theta$. The ZAMOs represent a generalization of the static observers in the Schwarzschild spacetime-this can be well demonstrated by the fact that the particles falling from rest at infinity remain purely radially falling relative to static observers in the Schwarzschild spacetimes and relative to LNRFs in the Kerr spacetimes [39]; for the principal null congruence (PNC) photons, i.e., purely radially moving photons, this property is, in Kerr spacetimes, realized in the Carter frames that differ slightly in comparison to the LNRFs [40].

Introducing the abbreviation

$$
A=\left(r^{2}+a^{2}\right)^{2}-a^{2} \Delta \sin ^{2} \theta
$$

the orthonormal tetrad of the LNRFs can be introduced as follows [41]

$$
\begin{aligned}
\omega^{r} & =(0, \sqrt{\Sigma / \Delta}, 0,0) \\
\omega^{\theta} & =(0,0, \sqrt{\Sigma}, 0) \\
\omega^{t} & =(\sqrt{\Delta \Sigma / A}, 0,0,0), \\
\omega^{r} & =\left(-\Omega_{\mathrm{LNRF}} \sqrt{A / \Sigma} \sin \theta, 0,0, \sqrt{A / \Sigma} \sin \theta\right) .
\end{aligned}
$$

The three-velocity of a particle having four-velocity $U^{\mu}$ has in the LNRFs the components $v^{i}$ given by the relation

$$
v^{i}=\frac{U^{(i)}}{U^{(t)}}=\frac{\omega_{\mu}^{(i)} U^{\mu}}{\omega_{\mu}^{(t)} U^{\mu}}
$$

where $i=r, \theta, \phi$. For the circular geodesic orbits, the only non-zero (axial) component reads [41]

$$
v^{\phi}=\frac{ \pm M^{1 / 2}\left(r^{2} \pm 2 a M^{1 / 2} r^{1 / 2}+a^{2}\right)}{\Delta^{1 / 2}\left(r^{3 / 2} \mp a M^{1 / 2}\right)} ;
$$

the upper sign determines the first family orbits (purely corotating in the black hole spacetimes), while the lower sign determines always the counter-rotating orbits. Recall that the first family stable circular orbits can become counter-rotating relative to the LNRFs (having $L>0$ ) around naked singularities with $a<3 \sqrt{3} / 4 \sim 1.3$, while the marginally stable circular orbit and slightly higher orbits have negative energy $E<0$ in the field of naked singularities with $a<\frac{4}{3} \sqrt{\frac{2}{3}} \sim 1.089$. For naked singularities with $a \rightarrow 1$, at the first family marginally stable orbits $E / m \rightarrow-\frac{1}{3} \sqrt{3}$ and efficiency of the Keplerian accretion reaches 1.5765 , while, for extreme black holes, it is only 0.4235-for details see [20].

\subsection{The Penrose Process}

The extraction of rotational energy of Kerr black holes from their ergosphere due to a very simple process of decay of an electrically neutral particle into two electrically neutral particles was proposed by R. Penrose in [1]. Denoting the incoming particle as particle 1, 
and the two particles resulting from the decay as 2 and 3, we can write the law of the (kinetic) four-momentum conservation in the form

$$
p_{\alpha(1)}=p_{\alpha(2)}+p_{\alpha(3)} .
$$

It is natural in astrophysically relevant situations to consider the incoming particle as incoming from infinity, and the simplest possibility is a particle falling from rest with $E_{1}=m_{1}$; another one is related to a particle infalling from the marginally stable circular geodesic assumed to be the inner edge of the Keplerian accretion disks. We can consider in the following only the equatorial motion of the particles; therefore, $p^{\theta}=0$ and only the axial and radial components of the motion remain non-zero. For our purposes, the energy conservation is crucial, namely, in the form given for the covariant energy

$$
E_{1}=E_{2}+E_{3} .
$$

If particle 2 has energy $E_{2}<0$ (having necessarily also $L_{2}<0$ [18]), then the third escaping particle has $E_{3}>E_{1}$ extracting rotational energy from the black hole-the mass and angular momentum of the Kerr black hole being decreased by the capture of the second particle.

The efficiency $\eta$ of the Penrose process relates the gained energy to the incoming energy due to the relation

$$
\eta=\frac{E_{3}-E_{1}}{E_{1}}=\frac{-E_{2}}{E_{1}} .
$$

The efficiency is strongest for the Penrose process in the equatorial plane when it is given for black holes by the relation

$$
\eta=\frac{1}{2}\left(\sqrt{r_{s} / r_{s p}}-1\right)
$$

with $r_{\mathrm{s}}=2$ representing the static limit radius at the equatorial plane and $r_{\mathrm{sp}}$ representing the splitting point located inside the ergosphere. In the case of the extreme Kerr black holes, we find the limiting value of $\eta_{\text {extr }}=\frac{1}{2}(\sqrt{2}-1) \sim 0.207$ for the splitting point approaching the black hole horizon.

We can thus see that the pure Penrose process is not extremely efficient, as its maximal efficiency $\eta \sim 0.207$ is only half of the efficiency of the Keplerian accretion around the extreme Kerr black hole that reaches $\eta \sim 0.427$. We expect that in the case of Kerr superspinars (naked singularities) the efficiency could be higher, being dependent on the unknown location of the superspinar surface-see [22,24,25].

To estimate the astrophysical relevance of the Penrose process realized in the equatorial plane, we have to calculate in the LNRF's velocity space $v^{r}-v^{\phi}$ the distance of the point corresponding to the incoming particle at the splitting point to the region of states with negative covariant energy. We thus have to relate the motion constants of particle 1 (and particle 2 assumed to be at any negative energy state) to the related velocities measured in the LNRF. We thus have to use the four-momentum in the LNRF taken in the form

$$
p^{\alpha}=m\left(\gamma, \gamma v^{i}\right)
$$

with the Lorentz factor

$$
\gamma=\left[1-\left(v^{r}\right)^{2}-\left(v^{\phi}\right)^{2}\right]^{-1 / 2} .
$$

The motion constants of the particle are related to the velocities due to the relations

$$
E=-p_{t}=-p_{(\alpha)} \omega_{t}^{(\alpha)}=\frac{m \gamma}{A^{1 / 2}}\left[r A^{1 / 2}+2 a v^{\phi}\right]
$$

and

$$
L=p_{\phi}=p_{(\alpha)} \omega_{\phi}^{(\alpha)}=m \gamma\left(\frac{A}{\Sigma}\right)^{1 / 2} v^{(\phi)} .
$$


The resulting schemes are represented for both Kerr black holes and Kerr naked singularities in Figure 2-see also [20,37]. We can see that the Penrose process is not astrophysically plausible around Kerr black holes because of the large gap between the velocities of the incoming particle and the particle with negative energy that is at least $\Delta v>0.5$ as demonstrated in [37]. On the other hand, in the case of Kerr superspinars (naked singularities), the Penrose process can be quite realistic, as the corotating circular geodesics directly enter the region of negative energy states, and even the marginally bound orbit with energy $E / m=1$ has the velocity gap $\Delta v<0.35$, if the superspinar dimensionless spin $a<1.089$ [20].
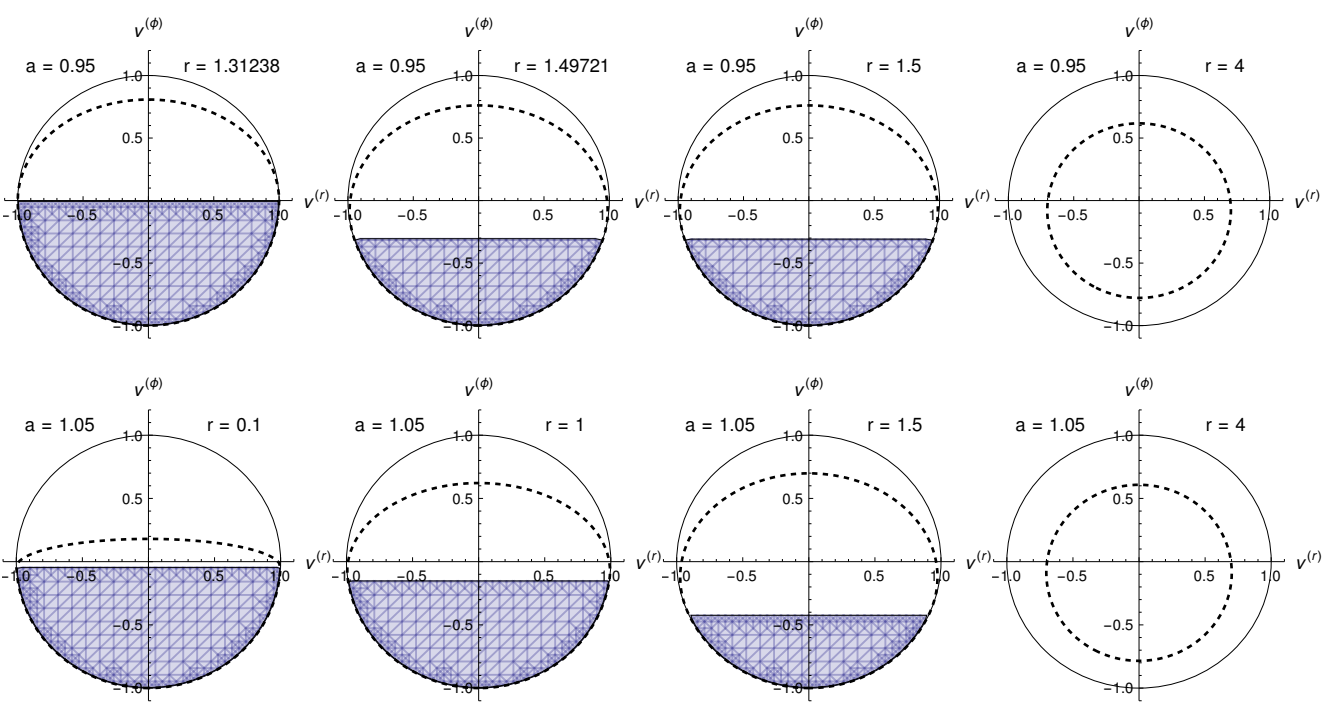

Figure 2. Distribution of different types of the particle equatorial motion in the LNRF velocity space $v^{(r)}-v^{(\phi)}$. Orbits from shaded regions have negative energy relative to infinity; the thick dashed curves represent last = bounded orbits. The black circle is given by the velocity norming condition. Notice the strong qualitative difference demonstrated in black-hole and naked-singularity spacetimes. The Penrose process is astrophysically plausible for rotating naked singularity because, for near extreme cases, the bound orbits can have negative energy relative to infinity.

\section{Magnetized Kerr Black Holes and Magnetic Penrose Process}

If the decay of charged particles is considered, it was shown that the magnetic Penrose process (MPP) around magnetized rotating black holes, i.e., Kerr black holes immersed in an external, large-scale magnetic fields, can have a much larger efficiency than the pure Penrose process, exceeding the annihilation value of $\eta=1[42,43]$.

\subsection{The Role of Magnetic Fields in Black Hole Physics}

In black hole physics, external magnetic fields play a very important role as known for a very long time [32]. The short-scale magnetic fields in the accretion disks generate viscosity and related transfer of matter and angular momentum in the radial direction due to the magneto-rotational instability $[7,44]$. The external (large scale) magnetic fields surrounding the rotating black holes can significantly influence ionized Keplerian disks, leading, in dependence on intensity of the electromagnetic interaction, to possible epicyclic oscillations of slightly charged matter that could be related to the high-frequency quasiperiodic oscillations (HF QPOs) observed in microquasars or active galactic nuclei $[26,45,46]$, or to an extraordinary manifestation of the MPP [1,42] creating ultra-high energy particles around magnetized supermassive rotating black holes [5,6,14,27,28]. Moreoever, there are indications that the Blandford-Znajek process [3] is a collective manifestation of the MPP [4]. Interesting new effects were discovered in relation to the radiation 0reaction due to synchrotron radiation of the strongly accelerated particles $[30,47]$. 
The circular orbits of charged particles located off the equatorial plane that occur around magnetized compact objects [48-50] indicate the existence of charged off-equatorial, "levitating," toroidal structures orbiting such objects. Contrary to the standard approach based on modeling the charged fluids in the "free-field" framework using an assumption of infinite conductivity $[2,3,51]$ that abandons the inertia of the fluid constituents, the opposite approximation of zero conductivity is assumed in the model of non-conducting tori that takes into account the inertia of the charged matter and that was developed in [52-54] - for an overview of this model, see [14]. It is interesting that the charged non-conducting tori can exist both as equatorial and off-equatorial structures or even as clouds on the rotational axis [53], being thus complementary to the equatorial multi-toroidal structures (ringed accretion disks) that can mix relatively counter-rotating tori, possibly created during evolution in active galactic nuclei [10-12,55-57]. Note that the low-density off-equatorial tori can be treated as collision-less plasma [58].

All the magnetic fields observed around compact objects can be considered as weak fields from the point of view of general relativity if their stress energy tensor is not strong enough to influence the spacetime curvature. The corresponding magnetic field intensity reads [59]

$$
B_{\mathrm{GR}}=10^{19}\left(\frac{M_{\odot}}{M}\right) G .
$$

In the present study giving a review of all the important variants of the Penrose process, we thus applied the pure Kerr geometry, as both the realistic electric charges [28] and magnetic fields (that are maximally $B_{G R}=10^{8} \mathrm{G}$ for stellar mass black holes and $B_{G R}=10^{5} G$ for supermassive black holes) have an insignificant influence on the spacetime geometry.

\subsection{Asymptotically Uniform Magnetic Field as Basic Approximation}

The external magnetic field in vicinity of the black hole horizon could be very complex, as shown in the magnetohydrodynamical general relativistic dynamical simulations (MHGRD) of magnetized toroidal structures [2]. However, the external magnetic field near the rotation axis of the tori, where the jets are located, can be well represented by a parabolic magnetic field or by the split-monopole field [3,51], and we can keep as the starting approximation the asymptotically uniform magnetic field introduced by Wald [29] that was applied in many astrophysical studies. To keep the symmetry of the background, it is useful to assume that the magnetic field lines are directed along the rotation axis of the geometry. (For the case of inclined magnetic field, see [60].)

The Wald field of intensity $B$, with lines oriented along the $z$-axis orthogonal to the geometry equatorial plane, is determined by the electromagnetic four-vector potential $A_{\alpha}$ with two non-zero components

$$
A_{t}=\frac{B}{2}\left(g_{t \phi}+2 a g_{t t}\right)-\frac{Q}{2} g_{t t}-\frac{Q}{2}, \quad A_{\phi}=\frac{B}{2}\left(g_{\phi \phi}+2 a g_{t \phi}\right)-\frac{Q}{2} g_{t \phi},
$$

with addition of the induced electric charge of the black hole $Q$ [29] — the maximal (Wald) value of the induced black hole charge reads $Q_{W}=2 a B$ (or $Q_{W}=2 a B M$ if we keep the mass term). For the Wald charge, the electromagnetic potential reduces to

$$
A_{t}=\frac{B}{2} g_{t \phi}-\frac{Q_{W}}{2}, \quad A_{\phi}=\frac{B}{2} g_{\phi \phi}
$$

It is very important that, even in this limiting case, the $A_{t}$ component remains nonzero, generating a possibly very strong acceleration mechanism in the vicinity of sufficiently massive black holes immersed in sufficiently strong magnetic fields. In the following, we 
concentrate on the case of the Wald charge $Q_{W}=2 a B$ representing the most plausible astrophysical situations. In the field of Kerr black holes, we then arrive to the formulae

$$
\begin{aligned}
& A_{t}=-a B M\left(\frac{r \sin ^{2} \theta}{\Sigma}+1\right) \\
& A_{\phi}=\frac{B \sin ^{2} \theta}{2}\left(r^{2}+a^{2}+\frac{2 M r a^{2}}{\Sigma} \sin ^{2} \theta\right) .
\end{aligned}
$$

The components of the electric and magnetic fields projected onto the tetrad of the LNRFs are generally given as $E_{(i)}=E_{\mu} e_{(i)}^{\mu}=F_{(i)(t)}$ for the electric field, and $B_{(i)}=B_{\mu} e_{(i)}^{\mu}=$ $\epsilon_{(i)(j)(k)} F^{(j)(k)}$ for the magnetic field. In the Kerr geometry and the related LNRFs, we thus obtain the following non-zero components [61]

$$
\begin{aligned}
& E_{(r)}=\frac{a M B}{\Sigma^{2} A^{1 / 2}}\left[2 r^{2} \Sigma \sin ^{2} \theta-\left(r^{2}+a^{2}\right)\left(r^{2}-a^{2} \cos ^{2} \theta\right)\left(1+\cos ^{2} \theta\right)\right], \\
& E_{(\theta)}=\frac{a M B \Delta^{1 / 2}}{\Sigma^{2} A^{1 / 2}} 2 a^{2} r \sin \theta \cos \theta\left(1+\cos ^{2} \theta\right), \\
& B_{(r)}=\frac{B \cos \theta}{\Sigma^{2} A^{1 / 2}}\left\{\left(r^{2}+a^{2}\right) \Sigma-2 M r a^{2}\left[2 r^{2} \cos ^{2} \theta+a^{2}\left(1+\cos ^{4} \theta\right)\right]\right\}, \\
& B_{(\theta)}=-\frac{B \Delta^{1 / 2} \sin \theta}{\Sigma^{2} A^{1 / 2}}\left[M a^{2}\left(r^{2}-a^{2} \cos ^{2} \theta\right)\left(1+\cos ^{2} \theta\right)+r \Sigma^{2}\right] .
\end{aligned}
$$

The twisting of magnetic lines caused by gravitational dragging generates a quadrupole electric field determined by the component $A_{t}$, and the "quadrupole" charge can be interpreted as the induced charge of the black hole [4,62]. Around extreme Kerr black holes, an analogy to the Meissner effect becomes relevant, canceling the induced charge, because such a black hole serves as a superconductor expelling the magnetic field lines [63].

For magnetic fields with a non-zero poloidal component, any observer can detect a non-zero electric field in the ergosphere $[29,64]$, as the induced electric charge could not be screened by plasma [65]. The total screening of the electric field needs the conditions

$$
\vec{B} . \vec{E}=0, \quad E^{2}-B^{2}<0
$$

satisfied simultaneously, where $\mathrm{E}$ and $\mathrm{B}$ are vectors of the electric and magnetic field as measured in the LNRFs [51]. However, $E^{2}-B^{2}$ is negative outside the ergosphere, but it is positive inside the ergosphere [51]. For this reason, the electric field generated by the combined effect of a uniform (generally poloidal) magnetic field and the black hole rotation is not screened inside the ergosphere where it can cause efficient acceleration of charged particles.

For estimation of the effectiveness of the acceleration process, the relation of the electromagnetic Lorentz force and the gravity, acting on a test particle with charge $q$ and mass $m$, influenced by a black hole with mass $M$, immersed in an uniform magnetic field of the strength $B$, we introduced a dimensionless "magnetic parameter" $\mathcal{B}[46,66]$

$$
\mathcal{B}=\frac{B}{2} \frac{q}{m} \frac{G M}{c^{4}} .
$$

In Table 1, we present a comparison of the relevance of various magnetic fields for various test particles including a charged dust grain (one electron lost, $m=2 \times 10^{-16} \mathrm{~kg}$ ). 
Table 1. Intensity of the magnetic field $B$ corresponding to the magnetic parameter $\mathcal{B}=1$ for various types of charged particles moving in the vicinity of the black hole of mass $M_{B H} \sim 10 M_{\odot}$.

\begin{tabular}{ccccc}
\hline $\boldsymbol{M}=\mathbf{1 0} \boldsymbol{M}_{\text {sun }} /$ & Electron & Proton & Fe+ & Charged Dust \\
\hline $\mathcal{B}=1$ & $10^{-3} \mathrm{G}$ & $4 \mathrm{G}$ & $240 \mathrm{G}$ & $10^{11} \mathrm{G}$ \\
\hline
\end{tabular}

There are several alternatives to the uniform magnetic field that are widely discussed in the astrophysical context. The first one is the dipole magnetic field that can be generated by circular current loops in the central plane of the black hole [67]. Second, in the vicinity of the black hole horizon, such a field can be well represented by the split-monopole approximation introduced in [3], where the approximation of the parabolic field was also introduced. Note that the parabolic fields are usually the outcomes of the numerical simulations of the toroidal structures treated in the framework of general relativistic magnetohydrodynamics [2]. Of special character is the electromagnetic field related to the Kerr-Newman black holes or their generalizations with the cosmological constant-see, e.g., [15]. For our purposes, the uniform magnetic field approximation can be quite sufficient [14].

\subsection{Motion of Charged Test Particles}

Charged test particle motion is determined by the Lorentz equation

$$
m \frac{D u^{\mu}}{D \tau}=e F_{v}^{\mu} u^{v}
$$

where $\tau$ is the proper time of the moving particle and $F_{v}^{\mu}$ is the Faraday tensor of the electromagnetic field. In the Kerr-Newman black hole backgrounds, the charged particle motion is fully regular, as the Lorentz equations can be separated and solved in terms of first integrals $[34,68]$ - in the magnetized Kerr black hole background, the motion is generally chaotic.

\subsubsection{Hamiltonian Formalism and Effective Potential of the Motion}

The symmetries of the considered magnetized Kerr black hole backgrounds imply the existence of two constants of the motion: energy $E$ and axial angular momentum $L$, which are determined by the conserved components of the canonical momentum

$$
-E=\pi_{t}=g_{t t} p^{t}+g_{t \phi} p^{\phi}+q A_{t}, \quad L=\pi_{\phi}=g_{\phi \phi} p^{\phi}+g_{\phi t} p^{t}+q A_{\phi} .
$$

To treat the motion, we used the Hamilton formalism. The Hamiltonian can be given as

$$
H=\frac{1}{2} g^{\alpha \beta}\left(P_{\alpha}-q A_{\alpha}\right)\left(P_{\beta}-q A_{\beta}\right)+\frac{1}{2} m^{2},
$$

where the generalized (canonical) four-momentum $P^{\mu}=p^{\mu}+q A^{\mu}$ is related to the kinematic four-momentum $p^{\mu}=m u^{\mu}$ and the electromagnetic potential term $q A^{\mu}$. The motion is governed by the Hamilton equations

$$
\frac{\mathrm{d} X^{\mu}}{\mathrm{d} \zeta} \equiv p^{\mu}=\frac{\partial H}{\partial P_{\mu}}, \quad \frac{\mathrm{d} P_{\mu}}{\mathrm{d} \zeta}=-\frac{\partial H}{\partial X^{\mu}}
$$

where the affine parameter and the particle proper time are connected as $\zeta=\tau / \mathrm{m}$. The Hamilton equations represent, in the general case, eight first-order differential equations that can be integrated numerically.

The combined gravitational and electromagnetic background of the magnetized Kerr black holes considered here is stationary and axially symmetric, and the related two constants of motion allow a reduction in the charged test particle motion to two-dimensional 
dynamics. Introducing the specific energy $\mathcal{E}=E / m$, the specific axial angular momentum $\mathcal{L}=L / m$, and the magnetic interaction parameter $\mathcal{B}=q B / 2 m$, the Hamiltonian reads

$$
H=\frac{1}{2} g^{r r} p_{r}^{2}+\frac{1}{2} g^{\theta \theta} p_{\theta}^{2}+H_{\mathrm{P}}(r, \theta) .
$$

We can define the effective potential of the radial and latitudinal motion that determines the energetic boundary for the particle motion $\left(H_{\mathrm{P}}=0\right)$, corresponding to turning points of the radial $\left(p^{r}=0\right)$ and the latitudinal $\left(p^{\theta}=0\right)$ motion. The energy condition implies for the effective potential the relation

$$
\mathcal{E}=V_{\text {eff }}(r, \theta)
$$

where

$$
V_{\mathrm{eff}}(r, \theta)=\frac{-\beta+\sqrt{\beta^{2}-4 \alpha \gamma}}{2 \alpha}
$$

with

$$
\begin{array}{r}
\beta=2\left[g^{t \phi}\left(\mathcal{L}-\tilde{q} A_{\phi}\right)-g^{t t} \tilde{q} A_{t}\right], \\
\alpha=-g^{t t}, \quad \gamma=-g^{\phi \phi}\left(\mathcal{L}-\tilde{q} A_{\phi}\right)^{2}-g^{t t} \tilde{q}^{2} A_{t}^{2}+2 g^{t \phi} \tilde{q} A_{t}\left(\mathcal{L}-\tilde{q} A_{\phi}\right)-1
\end{array}
$$

The effective potential defined here behaves well above the outer horizon; subtleties in the inner region of the Kerr geometry are discussed in [69]. The effective potential determines the allowed regions in the $r-\theta$ space for charged particles with fixed axial angular momentum-see Figure 3. It is crucial that the effective potential determines in a natural way the region where the magnetic Penrose process can be relevant, which is called the effective ergosphere. The boundary of the effective ergosphere is, for a charged particle with fixed axial angular momentum, determined by the relation

$$
\mathcal{E}=V_{\text {eff }}(r, \theta)=0 .
$$

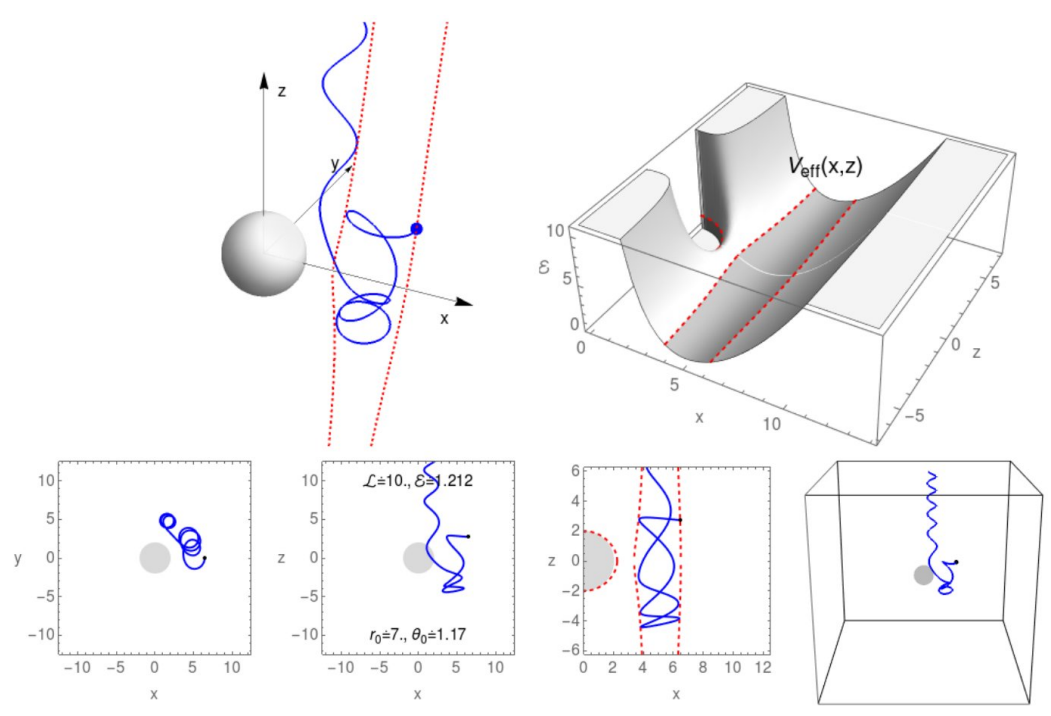

Figure 3. Effective potential of the charged particle motion and an example of the chaotic type of the particle motion.

Inside the effective ergosphere, the energy states with $E<0$ are possible; therefore, it is clearly the arena of the MPP. The effective ergosphere is not identical to the ergosphere, extension of which is independent of the details related to the particles, and it could significantly exceed the boundary of the ergosphere; in fact, there is no general limit in the Kerr spacetime except the event horizon. 
In the study of the MPP, a crucial role is played by the notion of ionized Keplerian disks. The standard Keplerian disks are considered as composed from matter that behaves as electrically neutral in external electromagnetic fields-the disk composed from a quasineutral mixture of electrons, protons, and ion behaves as collectively neutral matter if its density is sufficiently high to guarantee a sufficiently short mean free path of the particle motion in comparison with its orbital motion. However, near the edge of the disk, the density decreases significantly, and the particles start to feel the electromagnetic field as their mean free path increases to become comparable with extension of the orbital motion. (Note that an irradiation of the disk can also be caused by its ionization, as is discussed later.) The fate of the ionized part of the Keplerian disks is discussed in detail in [14]. One of the relevant cases, very important in the astrophysics of accretion disks, which occurs if the electromagnetic influence is sufficiently weak, is survival of this part of the disk in epicyclic regular motion, enabling explanation of HF QPOs observed in microquasars and active galactic nuclei [14].

\subsubsection{Chaotic Scattering}

Relevant for our purposes is the case of a strong electromagnetic field leading to destruction of the ionized part of the disk in the process of chaotic scattering [27]. To obtain a proper intuition on this phenomenon, we have to discuss the asymptotic behavior of the effective potential having the form [27]

$$
V_{\text {eff }}(x, z \rightarrow \infty)=2 a \mathcal{B}+\sqrt{1+\left(\frac{\mathcal{L}}{x}-\mathcal{B} x\right)^{2}} .
$$

The particles thus can reach infinity in the $z$-direction with minimal energy

$$
\begin{gathered}
\mathcal{E}_{\text {min }}=2 a \mathcal{B} \text { for } \mathcal{B} \geq 0, \\
\mathcal{E}_{\text {min }}=2 a \mathcal{B}+\sqrt{1-4 \mathcal{B L}} \text { for } \mathcal{B}<0 .
\end{gathered}
$$

Particles with energy $\mathcal{E} \geq \mathcal{E}_{\text {min }}$ can reach infinity, but their energy has to be modified there due to the "magnetic" factor to give $\mathcal{E}_{\infty}=\mathcal{E}-2 a \mathcal{B}$, because, for the motion in the homogeneous magnetic field, the effective potential at infinity in the flat spacetime is not containing the term $2 a \mathcal{B}$. In the asymptotically flat region of the magnetized Kerr black holes, we thus arrived in the cylindrical coordinates $t, \rho, \theta, \phi$ to the energy relation [27]

$$
\mathcal{E}_{\infty}^{2}=\left(\frac{\mathrm{d} z}{\mathrm{~d} \tau}\right)^{2}+\left(\frac{\mathrm{d} \rho}{\mathrm{d} \tau}\right)^{2}+g_{\phi \phi}\left(\frac{\mathrm{d} \phi}{\mathrm{d} \tau}\right)^{2}=\mathcal{E}_{z}^{2}+\mathcal{E}_{0}^{2}
$$

separation of the energy to the parts corresponding to the translational (longitudinal) motion

$$
\mathcal{E}_{z}^{2}=\left(\frac{\mathrm{d} z}{\mathrm{~d} \tau}\right)^{2}
$$

and the transverse (perpendicular) motion mixing the orbital and oscillatory radial motion

$$
\mathcal{E}_{0}^{2}=\left(\frac{\mathrm{d} \rho}{\mathrm{d} \tau}\right)^{2}+\left(\frac{\mathcal{L}}{\rho}-\mathcal{B} \rho\right)^{2}+1
$$

has been done. In the field of Kerr black holes, the motion constants are only $\mathcal{E}$ and $\mathcal{E}_{\infty}$, but conversion of the energies $\mathcal{E}_{z}$ and $\mathcal{E}_{0}$ is possible due to chaotic character of the motion [27].

In infinity, the particle state is characterized by the Lorentz factor

$$
\gamma=\frac{\mathrm{d} t}{\mathrm{~d} \tau}=\mathcal{E}_{\infty}
$$


and its part related to the longitudinal motion. The maximum value of the longitudinal Lorentz factor depends on the magnetic parameter and related velocity of the azimuthal motion [27]:

$$
\gamma_{z(\max )}=\mathcal{E}_{\infty}, u^{\phi}=0, \quad \text { for } \quad \mathcal{B}>0,
$$

and

$$
\gamma_{z(\max )}=\frac{\mathcal{E}_{\infty}}{\sqrt{1-4 \mathcal{B L}}}, \quad u^{\phi}=2 \mathcal{B L}, \quad \text { for } \quad \mathcal{B}<0 .
$$

For $\mathcal{B}>0$, full conversion of the transverse motion to the longitudinal motion is possible, corresponding to particles following the magnetic field lines. For $\mathcal{B}<0$, complete conversion is impossible, and a minimal Larmor orbital motion must be added to the translational motion-the minimum of axial angular velocity is given as $u^{\phi}=2 \mathcal{B L}$. The Lorentz factor can be of order of 10 for $\mathcal{B} \sim 1$, [27]; around realistic magnetized black holes, the Lorentz factor can take many orders of 10 [28].

We can conclude that fate of the ionized Keplerian disk is governed by the fight of gravity and electromagnetic forces that is reflected by the magnetic parameter $\mathcal{B}$. For $\mathcal{B} \ll 1$, gravity wins, and the character of the motion is close to the regular motion, while, for $\mathcal{B}$ $\sim 1$, there is the strong fight of the influences implying fully chaotic motion, and, for $\mathcal{B} \gg 1$, the electromagnetic forces win, the chaotic period of the motion is suppressed, and the regular motion is approached soon-see Figure 4 demonstrating the suppression of chaotic motion with increasing magnetic parameter.
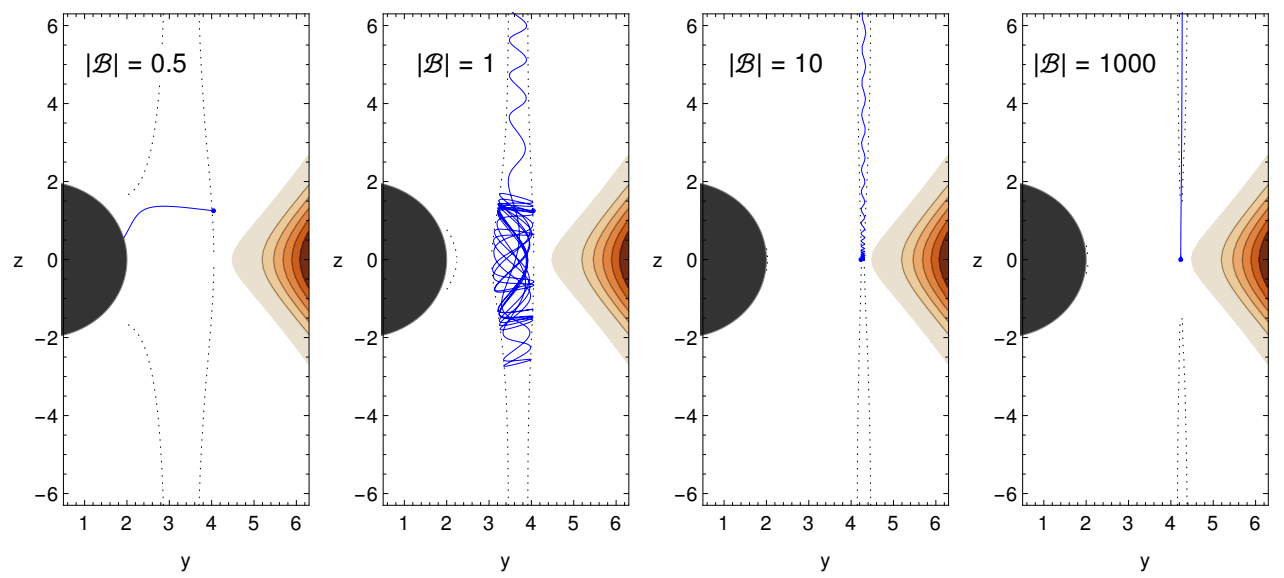

Figure 4. Charged particle chaotic scattering under the combined influence of rotating black hole and uniform magnetic field.

\subsection{Magnetic Penrose Process and Creation of Jets}

The energy balance of the MPP is determined by the local value of the electric part of the potential. We consider the MPP including the case of decaying charged particles or ionized Keplerian disks. For simplicity, we restricted attention to the equatorial motion, allowing for small impulsion to the vertical direction to enable the chaotic regime of the motion, allowing escape to infinity along the lines of the magnetic field. The energetic balance of the decay process was calculated in the equatorial plane where the highest efficiency of the MPP is obtained.

The effective potential of the equatorial motion around a magnetized Kerr black hole takes the form

$$
V_{\text {eff }}=-q A_{t}-\frac{g_{t \phi}}{g_{\phi \phi}} L+\left[-g_{t t}+\frac{g_{t \phi}^{2}}{g_{\phi \phi}}\left(\frac{L^{2}}{g_{\phi \phi}}+1\right)\right] ;
$$


due to the time component of the electromagnetic potential, the negative energy states are possible outside the ergosphere- the resulting effective ergosphere allows in dependence on $L$ the existence of negative energy states without any limit $[18,43]$.

We now consider that the first particle assumed to be generally positively charged (neutrality not excluded) splits onto two charged particles, the second one having a negative charge and the third one having a positive charge. Assuming that the second particle has a negative canonical energy $E_{2}<0$, the third one should have the canonical energy $E_{2}>E_{1}$ due to extraction of the black hole rotational energy caused by the capture of the second particle. Two conservation laws govern the process of the splitting process. The conservation of the electric charge

$$
q_{1}=q_{2}+q_{3}
$$

and the canonical momenta conservation

$$
P_{\alpha(1)}=P_{\alpha(2)}+P_{\alpha(3)} .
$$

The charged particle motion is characterized by its angular velocity related to the distant static observers

$$
\Omega=\frac{d \phi}{d t}=\frac{u^{\phi}}{u^{t}},
$$

restricted by the null vector limits on the four-momentum $p^{\alpha}, \Omega_{ \pm}$. The escaping third particle has the canonical conserved energy [28]

$$
E_{3}=\chi\left(E_{1}+q_{1} A_{t}\right)-q_{3} A_{t}
$$

with

$$
\chi=\frac{\Omega_{1}-\Omega_{2}}{\Omega_{3}-\Omega_{2}} \frac{X_{3}}{X_{1}}, \quad X_{i}=g_{t t}+\Omega_{i} g_{t \phi}
$$

where $\Omega_{i}\left(X_{i}\right)$ is the angular velocity (the velocity factor) of the $\mathrm{i}$-th particle. The MPP can work if the electromagnetic term $-q_{2} A_{t}$ plays a dominant role being positive- for $q_{3}>0$ and $B>0, a>0$ the condition $-q_{3} A_{t}<0$ is satisfied because of definition of $A_{t}$, and it will be dominant for large values of the magnetic parameter $\mathcal{B}$.

Under such conditions, the third particle (e.g., proton) energy $E_{3}=p_{t 3}+q A_{t}$ is very large, while the second particle energy $E_{2}=p_{t 2}-q A_{t}$ is negative with a very large magnitude (or vice-versa, due to the orientation of the magnetic field). Due to the chaotic scattering mechanism [27], the highly energetic third particle escapes to infinity along the magnetic field, and the second particle with large negative energy enters the black hole-see Figure 5.

The efficiency of the MPP can be expressed in the form

$$
\eta_{\mathrm{MPP}}=\chi-1+\frac{\chi q_{1} A_{t}-q_{3} A_{t}}{E_{1}}
$$

where all quantities are taken at the splitting point. As in the case of the the orbital motion of uncharged particles, for charged particles' motion, their angular velocity related to the static observers at infinity is restricted to the interval

$$
\Omega_{-} \leq \Omega \leq \Omega_{+}
$$

and the limiting cases determine the maximal allowed efficiency of the MPP process.

The MPP can work in two regimes having significantly different characters and efficiencies-regimes of moderate and ultra-high efficiency. The original Penrose process involving only electrically neutral particles represents a third, low-efficiency regime with $\eta_{\mathrm{PP}(\max )} \sim 0.207$ that is lower than the efficiency of the Keplerian accretion process for 
near-extreme Kerr black holes $(\eta \sim 0.427)$ [41] or the near-extreme Kerr naked singularities $(\eta \sim 1.573)[20,21]$.
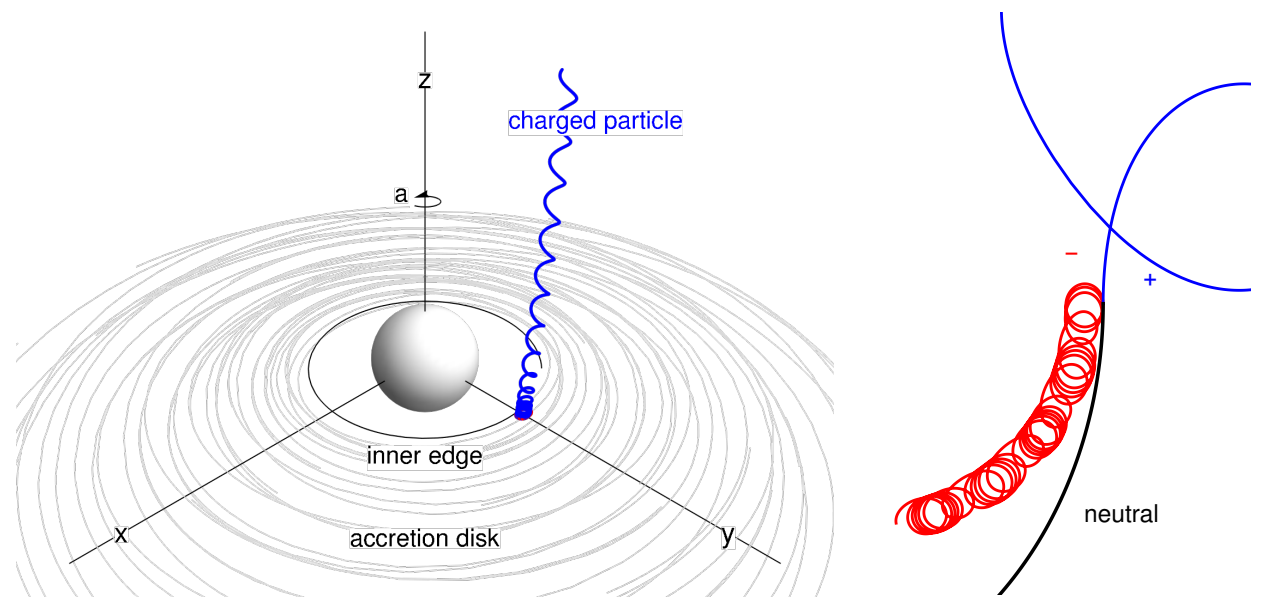

Figure 5. Magnetic Penrose Process for ionized neutral particle. The positively charged particle (proton) escapes into infinity, gaining positive energy from the black hole, while the negatively charge particle (electron) is captured by the black hole, loading to it negative energy.

\subsubsection{Moderately Efficient Regime of Mpp}

The moderate regime of the MPP corresponds to the electrically charged incoming particle in a situation where the electromagnetic forces are dominant, i.e., the condition $\left|\frac{q}{m} A_{t}\right|>>\left|u_{t}\right|$ is satisfied. In the moderate regime, the efficiency reads [5,14]

$$
\eta_{\mathrm{MPP}}^{\mathrm{mod}} \sim \frac{q_{3}}{q 1}-1
$$

while the condition $q_{3}>q_{1}$ is satisfied. The efficiency of the MPP process in this regime is thus estimated as $\eta_{\mathrm{MPP}}^{\mathrm{mod}} \sim 1$ and can reach several units, being thus significantly larger than the efficiency of the original Penrose process related to neutral particles. In fact, it was shown for the magnetically arrested accretion disks that the efficiency of the MPP is $\eta \sim 3$ [70], which s in agreement with the moderate MPP.

In the MPP moderate regime, the electric field induced by the black hole rotation is successively neutralized by its operation. The moderate regime of the MPP is related to the Blandford-Znajek process [3] that is considered as a collective action of the MPP process. Both the MPP and Blandford-Znajek processes are driven by the quadrupole electric field generated by the magnetic field lines twisted due to the spacetime dragging [4]. The Blandford-Znajek process as a collective phenomenon is caused by the difference of the electric field generated by the spacetime dragging of the magnetic field at the equatorial plane, where the induced electric field is maximal, and the pole where it is minimized-the potential difference is then feeding the related electric currents. Of course, the BlanfordZnajek process requires a very high threshold intensity of the external magnetic field that enables ionization of the vacuum by generation of the electron-positron pairs-the threshold magnetic intensity is given by the relation $[3,4]$

$$
B_{\text {thre }} \approx 6.2 \times 10^{4}\left(\frac{M}{a}\right)^{3 / 4}\left(\frac{10 M_{\odot}}{M}\right)^{1 / 2} G
$$

that is well met for both the stellar mass black holes where $B \sim 10^{8} \mathrm{G}$ and supermassive black holes in active galactic nuclei where $B \sim 10^{4} G$. Moreover, the Blanford-Znajek process also assumes near-extreme Kerr black holes. On the other hand, a more general MPP process can work at a much lower intensity of the magnetic field (even $B \sim m G$ is sufficient) and is not requiring fast-rotating black holes. 


\subsubsection{Extremely Efficient Regime of Mpp}

The highly efficient regime of the MPP works for the ionization of neutral matter, and its efficiency is dominated by the electromagnetic component

$$
\eta_{\mathrm{MPP}}^{\mathrm{extr}} \sim \frac{q_{3}}{m_{1}} A_{t} .
$$

In the extreme regime, the efficiency can be as large as $\eta_{\mathrm{MPP}}^{\text {extr }} \sim 10^{12}$ for sufficiently large magnetic fields and sufficiently supermassive Kerr black holes.

It is very useful to demonstrate the differences in the efficiency of the moderate and extreme MPP, making comparisons in very similar situations. For these purposes, we considered two similar splittings near a magnetized Kerr black hole having $M=10 M_{\odot}$, $a=0.8$, and $B=10^{4} \mathrm{G}$, due to an electron loss by a charged and uncharged Helium atom:

$$
\mathrm{He} \rightarrow \alpha\left(\mathrm{He}^{++}\right)+2 e^{-}, \quad \mathrm{He}^{+} \rightarrow \alpha\left(\mathrm{He}^{++}\right)+e^{-} .
$$

The estimate on the efficiency for the extreme MPP gave

$$
\eta_{\mathrm{He}}^{\mathrm{extr}} \sin 2.4 \times 10^{3},
$$

and for the moderate MPP we obtained

$$
\eta_{\mathrm{He}^{+}}^{\bmod } \sim 1
$$

We thus immediately see that for the split charged particle, we obtained efficiency of the order of 1 , but, for the electrically neutral particle, the efficiency reached an order of $10^{3}$. We thus naturally expect that for supermassive black holes of mass $M \sim 10^{10} M_{\odot}$, in the field having $B \sim 10^{4} G$, the efficiency can reach values $\eta_{\mathrm{MPP}}^{\text {extr }} \sim 10^{12}$ [28], corresponding to protons accelerated up to the velocities with Lorentz factor $\gamma \sim 10^{12}$. Of course, in the extreme regime of the MPP, the question of the energy gap to the negative energy states, important in the original Penrose process, is irrelevant, as the magnetic field present at the ionization point is the agent immediately acting to put the second particle into the state with negative energy relative to distant observers.

The crucial aspect of the MPP extreme regime is the neutrality of the first (incoming) particle that could reach the vicinity of the horizon, unavailable to charged particles, where the acceleration can be efficient-simultaneously, the space can be free of matter there, enabling thus the escape of the accelerated particle to infinity. Of course, the ionized Keplerian disks fulfill well these conditions. In the MPP related to ionized Keplerian disks, we can write

$$
\begin{array}{r}
P_{\alpha(1)}=P_{\alpha(2)}+P_{\alpha(3)}, \quad p_{\alpha(1)}=p_{\alpha(2)}+q A_{\alpha}+p_{\alpha(3)}-q A_{\alpha \prime} \\
m_{(1)} \geq m_{(2)}+m_{(3)}, \quad 0=q_{(2)}+q_{(3)} .
\end{array}
$$

Assuming that the mass of the second particle is much smaller than the mass of the third particle,

we can put the restriction

$$
m_{(1)} \sim m_{(2)} \gg m_{(3)}
$$

$$
p_{\alpha(1)} \sim p_{\alpha(3)} \gg p_{\alpha(2)} .
$$

In the ionized Keplerian disks, the splitting electrically neutral particle follows (nearly) circular geodesic orbits, so we can assume the third particle escaping with large canonical energy $E_{(3)}=p_{t(3)}-q_{(3)} A_{t}$, while the second particle is captured with large negative energy $E_{(2)}=p_{t(2)}-q_{(2)} A_{t}=p_{t(2)}+q_{(3)} A_{t}$.

Moreover, the chaotic scattering transmutes the original nearly circular motion of the ionized Keplerian disks to the linear motion of scattered particles along the magnetic field lines. The extreme MPP thus could model (in addition to the Blanford-Znajek model) the 
creation of strongly relativistic jets observed in active galactic nuclei. The external magnetic field plays the role of a catalyst of the acceleration of the charged particles generated by the ionization - extraction of the black hole rotational energy occurs due to captured negative-energy-charged particles. The magnetic field lines then collimate the motion of accelerated charged particles. Under the inner edge of the Keplerian disk, an under-dense region is located, where the charged particles acquire the highest possible energy due to acceleration by the strongest potential difference, and they could survive their travel to distant observers, if kept by the magnetic field close to the black hole rotation axis where the lowest density of the jets is expected.

In the vicinity of the horizon, the splitting process in the equatorial plane implies the efficiency of the extreme MPP taking the form (now in the standard units)

$$
\eta_{\mathrm{MPP}}^{\mathrm{extr}} \sim \frac{q_{3} G B M a}{m_{1} c^{4}}\left(1-\frac{r_{s}}{2 r_{s p}}\right)
$$

where $r_{s}=2 G M / c^{2}$ is the static limit radius (boundary of the ergosphere) at the equatorial plane, and $r_{s p}$ is the splitting point radius that can be potentially outside the ergosphere. The efficiency is governed by the electromagnetic acceleration - it exceeds the "annihilation" value of $\eta=1$ for electrons accelerated around a stellar mass of black holes immersed in the field with $B \sim m G$.

For a Keplerian disk ionized around a non-rotating black hole, the MPP generates winds not able to escape to infinity, as they can have only energy from the rotational energy of the orbiting matter extracted due to the chaotic scattering (similarly to the Payne-Blandford process [71]).

\subsubsection{Ultra-High Energy Cosmic Rays as Products of Mpp in the Extreme Regime}

The cosmic rays are high-energy protons or ions, demonstrating an isotropic distribution that can be explained only by their extra-galactic origin. The ultra-high-energy cosmic rays (UHECRs) are particles with energy $E>10^{18} \mathrm{eV}$-particles exceeding $E>10^{21} \mathrm{eV}$ are rarely observed and are of high interest as they overcome the GZK limit $\left(10^{19} \mathrm{eV}\right)$ caused by interactions with the cosmic microwave background.

The energy loss determined by the GZK-cutoff puts strong limits on the distance of sources of the cosmic rays with energy overcoming the GYK limit-the corresponding restricting distance is estimated as $l \sim 100 \mathrm{Mpc}[72,73]$. The observations give the correlation of the ultra-high energy particles with $E>10^{20} \mathrm{eV}$ to the active galactic nuclei at distances lower than $100 \mathrm{Mpc}$ [7].

The maximum of the energy of a charged particle generated in the extreme regime of the MPP is given (in physical units) as

$$
E_{\mathrm{MPP}}=1.3 \times 10^{21} \mathrm{eV} \frac{q}{e} \frac{m_{p}}{m} \frac{a B}{10^{4} G} \frac{M}{10^{10} M_{\odot}} .
$$

This dependence is illustrated in Figure 6. We can see that protons with energy $E>10^{21} \mathrm{eV}$ are generated by mildly spinning $(a \sim 0.8)$ supermassive black holes with mass $M=10^{10} M_{\odot}$, in the magnetic field with $B=10^{4} G$. The maximum energy of ions generated under the same conditions as protons is lowered by the factor corresponding to the specific charge of the considered particles.

The galaxy center SgrA* black hole, being the closest supermassive black hole with mass $M_{\mathrm{SgrA} *} \sim 4.14 \times 10^{6} M_{\odot}$ [74], spin $a_{\mathrm{SgrA} *} \sim 0.5$ [75], and the magnetic field intensity $B \sim 10 \mathrm{G}$ [76] should accelerate frequently observed particles due to its special position and shortest distance. The predicted maximal energy of protons generated near the horizon of SgrA ${ }^{*}$ black hole $E_{\mathrm{p}-\mathrm{SgrA} *}=10^{15.6} \mathrm{eV}$ is very interesting from this point of view as it corresponds to the knee of the energy spectrum in the observed data, located at $E_{\text {knee }} \sim 10^{15.6} \mathrm{eV}$, where the observed particle flux is significantly suppressed, which is in agreement with assumed existence of a strong single source at short distance. Moreover, 
the maximal proton energy $E_{\mathrm{MPP}} \sim 10^{19} \mathrm{eV}$ can be related to the M87 galaxy supermassive black hole with $M=7 \times 10^{9} M_{\odot}$ and $B=10^{2}$.
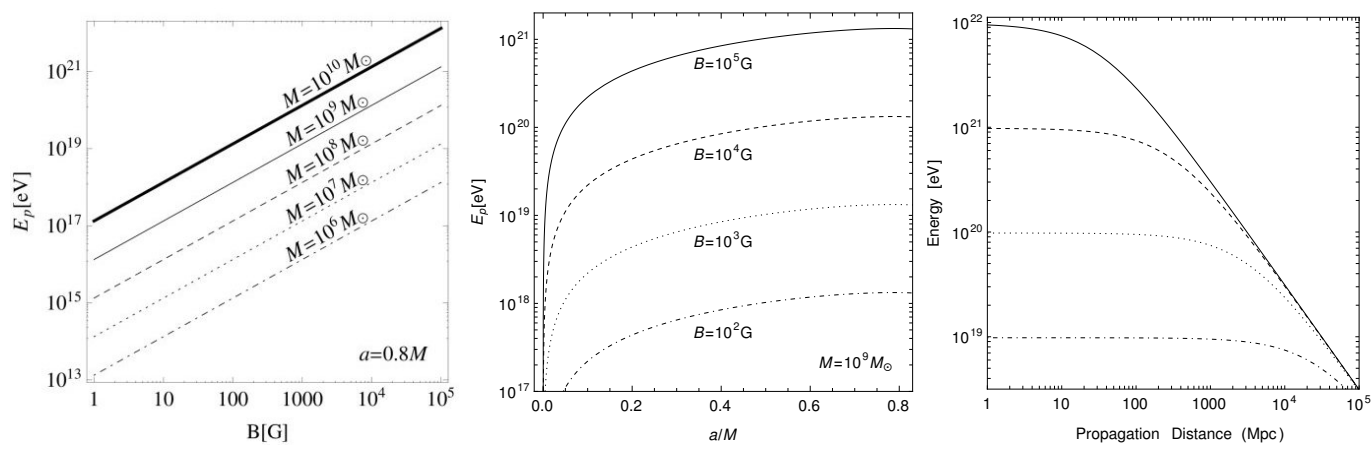

Figure 6. Escaping particles with increasing energy due to increasing magnetic parameter. Suppression of the periodic interval of the chaotic motion increases with increasing magnetic parameter.

If the maximal energy $E \sim 10^{21} \mathrm{eV}$ is related to protons, then in the same source we have to expect electrons accelerated up to energy $10^{24} \mathrm{eV}$ because of the factor of $m_{p} / m_{e} \sim 1820$-however, nothing like that is observed. The explanation is hidden in the efficiency of the deceleration (energy damping) of the charged particles due to back-reaction onto their electromagnetic (synchrotron) radiation in the magnetic field near the black hole. To the energy $E \sim 10^{21} \mathrm{eV}$, protons near the rotating magnetars are accelerated, as the magnetar mass decrease of 10 orders to $M \sim 1 M_{\odot}$ is just compensated by an increase in the magnetic strength to $B \sim 10^{14} \mathrm{G}$. Again, nothing like that is observed-the reason is again the back-reaction on the radiation. The back-reaction due to the radiation self-force is thus extremely important in connection to the particle acceleration and their observations; so we discuss this point carefully.

\subsubsection{Synchrotron Radiation of Accelerated Charged Particles}

The charged particles (protons, ions, or electrons) accelerated to ultra-high energy can be detected by distant observers, if the role of the back-reaction force is small or negligible. Therefore, we discuss the charged particle motion considering both the standard Lorentz force and the radiation reaction force $f_{R}^{\mu}$. In the non-relativistic limit, the synchrotron radiation generates the back-reaction force $f_{R}=\frac{3 q^{2}}{2 m} \frac{d^{2} u^{\alpha}}{d \tau^{2}}$ orthogonal to the four-velocity, satisfying thus the relation $f_{R}^{\mu} u_{\mu}=0$. Its covariant form reads [77]

$$
f_{R}^{\mu}=\frac{2 q^{2}}{3 m}\left(\frac{d^{2} u^{\mu}}{d \tau^{2}}+u^{\mu} u_{v} \frac{d^{2} u^{v}}{d \tau^{2}}\right) .
$$

In general, the Lorentz-Dirac equations take the form [30]

$$
\frac{D u^{\mu}}{d \tau}=\tilde{q} F^{\mu}{ }_{v} u^{v}+\tilde{q} \mathcal{F}^{\mu}{ }_{v} u^{v} .
$$

The first term on the right-hand side of Equation (83) is the Lorentz force that is determined by the electromagnetic tensor $F_{\mu \nu}$ of the external electromagnetic field, while the second term is the back-reaction self-force determined by the radiative field $\mathcal{F}_{\mu \nu}=$ $\mathcal{A}_{v, \mu}-\mathcal{A}_{\mu, v}$. The vector potential of the self-electromagnetic field is governed by

$$
\square \mathcal{A}^{\mu}-R^{\mu}{ }_{v} \mathcal{A}^{v}=-4 \pi j^{\mu},
$$


where $\square=g^{\mu v} D_{\mu} D_{v}$ is the Laplace operator, $D_{\mu}$ is the covariant differentiation, and $R_{\nu}^{\mu}$ is the Ricci tensor of the spacetime. The self-field is determined by the potential given by the retarded solution of Equation (84)

$$
\mathcal{A}^{\mu}(x)=q \int G_{+\lambda}^{\mu}(x, z(\tau)) u^{\lambda} d \tau
$$

$G_{+\lambda}^{\mu}$ is the retarded Green function. The integration in determining the self-field potential is considered for the particle worldline $z(\tau)$, and the four-velocity $u^{\mu}(\tau)=$ $d z^{\mu}(\tau) / d \tau[78]$.

The general relativistic form of the radiating charged particle dynamics takes the form $[79,80]$

$$
\begin{aligned}
& \frac{D u^{\mu}}{\mathrm{d} \tau}=\tilde{q} F^{\mu}{ }_{\nu} u^{v}+\frac{2 q^{2}}{3 m}\left(\frac{D^{2} u^{\mu}}{d \tau^{2}}+u^{\mu} u_{v} \frac{D^{2} u^{v}}{d \tau^{2}}\right) \\
& +\frac{q^{2}}{3 m}\left(R^{\mu}{ }_{\lambda} u^{\lambda}+R^{v}{ }_{\lambda} u_{v} u^{\lambda} u^{\mu}\right)+\frac{2 q^{2}}{m} f_{\text {tail }}^{\mu v} u_{v} ;
\end{aligned}
$$

the last term of Equation (86) giving the tail integral reflecting the role of back-scattering of the radiation, which is determined by the Green function $[78,80]$

$$
f_{\text {tail }}^{\mu \nu}=\int_{-\infty}^{\tau-0^{+}} D^{[\mu} G_{+\lambda^{\prime}}^{\nu]}\left(z(\tau), z\left(\tau^{\prime}\right)\right) u^{\lambda^{\prime}} d \tau^{\prime} .
$$

As the Ricci tensor vanishes in the vacuum Kerr spacetime, the related terms are irrelevant. Similarly, it can be demonstrated that the tail integral is irrelevant in our considerations $[14,30,81,82]$. Therefore, the radiation reaction force takes the form

$$
f_{R}^{\mu}=\frac{2 q^{2}}{3 m}\left(\frac{D^{2} u^{\mu}}{d \tau^{2}}+u^{\mu} u_{v} \frac{D^{2} u^{v}}{d \tau^{2}}\right)
$$

and the Lorentz-Dirac equation reads

$$
\frac{D u^{\mu}}{d \tau}=\tilde{q} F^{\mu} u^{v}+f_{R}^{\mu}
$$

The Lorentz-Dirac equation has a weak point as it gives the runaway solutions [30] - this disease can be cured by lowering the order of the differential equations $[14,30]$. Reducing the second derivative of the four-velocity, we arrive to the self-force expressed in the form

$$
f_{R}^{\alpha}=k \tilde{q}\left(\frac{D F_{\beta}^{\alpha}}{d x^{\mu}} u^{\beta} u^{\mu}+\tilde{q}\left(F_{\beta}^{\alpha} F^{\beta}{ }_{\mu}+F_{\mu v} F_{\sigma}^{v} u^{\sigma} u^{\alpha}\right) u^{\mu}\right)
$$

corresponding to the covariant form of the Landau-Lifshitz equations.

Detailed analysis of the motion of charged particles around a magnetized Schwarzschild black hole was presented in [30]; the widening of circular orbits was discussed in [31]. Examples of the role of the self-force on the motion around a magnetized Kerr black hole can be found in [14] on page 56. The synchrotron radiation has been studied also in $[83,84]$ using a covariant form of the flat space results and recently in [85] —however, without inclusion of the radiation reaction force.

For our purposes, the calculation of the energy loss is crucial. For the equatorial motion, the energy loss is given by the relation [30]

$$
\frac{d \mathcal{E}}{d \tau}=-2 k \mathcal{B}\left[2 \mathcal{B} \mathcal{E}^{3}-\mathcal{E}\left(2 \mathcal{B} f+\frac{u^{\phi}}{r}\right)\right] .
$$


For the ultra-high-energy particles $(\mathcal{E} \gg 1)$, the most significant contribution to the energy loss is given by the first term in square brackets of (91). The energy loss is related to the relaxation time $\tau$ required for decay of the radial oscillatory motion of a charged particle. The rate of the energy loss is related to the relaxation time as

$$
\dot{\mathcal{E}}=\frac{\mathcal{E}_{f}-\mathcal{E}_{i}}{\tau},
$$

where $\mathcal{E}_{i}\left(\mathcal{E}_{f}\right)$ denote the initial (final) energy of the particle. For ultrarelativistic particles, the energy loss reads

$$
\frac{d \mathcal{E}}{d \tau}=-4 \mathcal{B}^{2} k \mathcal{E}^{3}
$$

giving the solution

$$
\mathcal{E}(\tau)=\frac{\mathcal{E}_{i}}{\sqrt{1+8 \mathcal{B}^{2} k \mathcal{E}_{i}^{2} \tau}},
$$

with $\mathcal{E}_{i}$ denoting the initial energy. The relaxation time $\tau$ can be expressed as [30]

$$
\tau=\frac{1}{4 k \mathcal{B}^{2}} \frac{\mathcal{E}_{i}^{2}-\mathcal{E}_{f}^{2}}{\mathcal{E}_{i}^{2} \mathcal{E}_{f}^{2}} .
$$

For large values of $\mathcal{B}$, we arrive to the simple form [14]

$$
\tau_{\max } \approx \frac{1}{k \mathcal{B}^{2} f(r)}, \quad \mathcal{B} \gg 1
$$

enabling a fast estimation of the relevance of the self-force effects in connection to realistic astrophysical scenarios. We thus have to relate the particle and background parameters to the relaxation time.

For the characteristic values of the magnetic fields near the stellar mass $\left(M \sim 10 M_{\odot}\right.$, $\left.B \sim 10^{8} \mathrm{G}\right)$ and supermassive black holes $\left(M=10^{9} M_{\odot}, B \sim 10^{4} \mathrm{G}\right)[86,87]$, we find for electrons

$$
\begin{aligned}
\mathcal{B}_{\mathrm{BH}} & \approx 4.32 \times 10^{10} \text { for } M=10 M_{\odot}, \\
\mathcal{B}_{\mathrm{SMBH}} & \approx 4.32 \times 10^{14} \text { for } M=10^{9} M_{\odot} .
\end{aligned}
$$

For protons, the values of $\mathcal{B}$ in (97) and (98) decrease by the factor $m_{p} / m_{e} \approx 1836$. The extremely large values of $\mathcal{B}$ imply a strong role of magnetic fields in charged particles dynamics in realistic astrophysical scenarios.

The influence of the radiation reaction force on the energy damping, represented by the relaxation time $\tau$, depends strongly on the parameter combining the particle and the black hole characteristics-the parameter $k$ is expressed in dimensionless form as

$$
k=\frac{2}{3} \frac{q^{2}}{m G M} .
$$

The parameter $k$ governs strongly the realistic astrophysical scenarios, although it is very small, much lower than $\mathcal{B}$. For example, we find for electrons orbiting stellar mass and supermassive black holes

$$
\begin{aligned}
k_{\mathrm{BH}} & \sim 10^{-19} \quad \text { for } M=10 M_{\odot}, \\
k_{\mathrm{SMBH}} & \sim 10^{-27} \text { for } M=10^{9} M_{\odot} .
\end{aligned}
$$

For protons orbiting the same object as electrons, $k$ decreases by the factor $m_{p} / m_{e} \approx$ 1836 , as for $\mathcal{B}$.

The parameter $k$ is very low in relation to the parameter $\mathcal{B}$, but the particle energy damping can be very strong, as the relaxation time depends quadratically o a $\mathcal{B}$ that is large 
for realistic magnetized black holes. In Table 2, the relaxation time for electrons and protons is given for the same conditions around magnetized black holes. The relaxation times have to be confronted with the orbital timescales $\tau_{c}$; particles orbiting at the ISCO imply

$$
\begin{aligned}
\tau_{c} & \sim 10^{-3} \mathrm{~s}, \quad \text { for } M=10 M_{\odot}, \\
\tau_{c} & \sim 10^{4} \mathrm{~s}, \quad \text { for } M=10^{9} M_{\odot},
\end{aligned}
$$

For Sgr A* supermassive black holes, we find the electron decay time $\sim 10^{4} \mathrm{~s}$, while the ISCO orbital time is $\sim 10^{3} \mathrm{~s}$, being by one order smaller that the decay time.

Table 2. Energy decay times of electrons $\left(\tau_{e}\right)$ and protons $\left(\tau_{p}\right)$ orbiting a black hole immersed in a uniform magnetic field with values of $B$ characteristic for various astrophysical situations.

\begin{tabular}{ccc}
\hline B (Gauss) & $\tau_{\boldsymbol{e}}(\mathbf{s})$ & $\tau_{\boldsymbol{p}}(\mathbf{s})$ \\
\hline $10^{15}$ & $10^{-22}$ & $10^{-12}$ \\
$10^{8}$ & $10^{-8}$ & $10^{2}$ \\
$10^{4}$ & 1 & $10^{10}$ \\
1 & $10^{8}$ & $10^{1}$ \\
$10^{-5}$ & $10^{18}$ & $10^{28}$ \\
\hline
\end{tabular}

The relaxation time due to the charged particle oscillatory motion can be estimated by the relation [14]

$$
\tau \sim \frac{m^{3}}{q^{4} B^{2}}
$$

depending cubically on the particle mass and quadratically on the magnetic field intensity. Typical relaxation decay times of electrons and protons are given in Table 2.

Since $m_{p} / m_{e} \approx 1836$, the ratio of relaxation times of proton to electron, at fixed conditions, is very large, $\tau_{p} / \tau_{e} \sim 10^{10}$, in correspondence with the factor of $\left(m_{p} / m_{e}\right)^{3} \sim 10^{10}$. For this reason, the energy decay of electrons is relevant around magnetized black holes with plausible magnetic fields giving ultra-high energetic particles, so that electrons are significantly slowed and can not be observed as UHECR. The energy decay of protons (and ions) is irrelevant around magnetized black holes accelerating ultra-high energetic particles, and such energetic protons can also keep their energy on the distances $\sim 100 \mathrm{Mpc}$ comparable to the GZK limiting distance-we thus can observe them as UHECR. Simply saying, under fixed conditions, electrons are accelerated with efficiency $\sim 10^{3}$ larger than protons, but efficiency of their energy decay is $\sim 10^{10}$ larger than for protons. On the other hand, the energy due to acceleration by a given electromagnetic field depends linearly on $B$, but energy decay caused by the radiative reaction force depends on $B^{2}$; for protons, the energy decay is relevant exclusively around magnetars.

Charged particles (e.g., protons) can be accelerated to the same energy around magnetized supermassive black holes with $M \sim 10^{10} M_{\odot}, B \sim 10^{5} G$, and magnetars with $M \sim M_{\odot}$, $B \sim 10^{15} \mathrm{G}$, but around magnetars, the particle energy decays with efficiency $10^{10}$ higher than around the magnetized supermassive black hole. Therefore, there are no extremely energetic particles coming from magnetars, but we can see protons (ions) coming from magnetized supermassive black holes.

The play of the MPP acceleration and related energy decays at fixed conditions around a magnetized black hole, along with the energy decay related to the intergalactic travel of the ultra-high energy protons and ions, could help in localization of the active galatic nuclei emitting such particles.

For example, the calculations of energy decay of particles with $E>10^{20} \mathrm{eV}$, traveling across very weak magnetic field of $B \sim 10^{-5} \mathrm{G}$ representing the intergalactic magnetic field, demonstrate that particles with energy $E<10^{21} \mathrm{eV}$ can survive the distance $l \sim 100 \mathrm{Mpc}$ comparable to the GZK limit, but particles with energy $E \sim 10^{22} \mathrm{eV}$ can survive at the distance $l \sim 10 \mathrm{Mpc}$ [28]. 


\section{Electric Penrose Process}

The charge is one of the three characteristics allowed by the no-hair theorem (along with the mass and spin) to determine the most general black holes [18]. However, in astrophysics, the black hole charge is often neglected because of non-plausibly large charges necessary for the Reissner-Nordstrom spacetimes. On the other hand, we know that the electric charge can occur at a black hole because of the induction of electric field due to the magnetic field lines dragged by the Kerr black hole spacetime in the Wald solution [29], or in more general situations discussed, e.g., in $[3,4,14,28,38,51]$. Moreover, a small hypothetical electric charge could appear even in a non-rotating Schwarzschild black hole generating a test electric field whose influence on the black hole spacetime structure can be quite abandoned, but its role in the motion of test charged particles could be very strong $[88,89]$.

Because of the proton-to-electron mass ratio, the balance of the gravitational and Coulombic forces for the particles close to the horizon is reached when the black hole acquires a positive net electric charge $Q \sim 3 \times 10^{11} \mathrm{Fr}$ per solar mass [88]. Matter around the black hole can be also ionized by irradiating photons causing escape of electrons [90] - the positive charge of the black hole is then $Q \sim 10^{11} \mathrm{Fr}$ per solar mass. (In the Wald mechanism related to the magnetic field lines dragged by the black hole rotation [14,29], both the black hole and surrounding magnetosphere acquire opposite charges of the same magnitude $Q \sim 10^{18} \mathrm{Fr}$.) The realistic value of the black hole charge may for these reasons vary in the interval

$$
10^{11} \frac{M}{M_{\odot}} \text { Fr } \lesssim Q_{\mathrm{BH}} \lesssim 10^{18} \frac{M}{M_{\odot}} \text { Fr. }
$$

It is naturally interesting to know if an electric Penrose process is allowed in the circumstances corresponding to matter ionized in the vicinity of electrically charged black holes-it was demonstrated in [91] that relevant acceleration is really possible; we summarize the results.

\subsection{Charged Particles around Weakly Charged Schwarzschild Black Hole}

The Schwarzschild spacetime is governed by the line element

$$
d s^{2}=-f(r) d t^{2}+f^{-1}(r) d r^{2}+r^{2}\left(d \theta^{2}+\sin ^{2} \theta d \phi^{2}\right),
$$

where $f(r)$ is the lapse function containing the black hole mass $M$

$$
f(r)=1-\frac{2 M}{r} .
$$

The radial electric field corresponding to the small electric charge $Q$ is represented by the only non-zero covariant component of the electromagnetic four-potential $A_{\mu}=\left(A_{t}, 0,0,0\right)$ having the Coulombian form

$$
A_{t}=-\frac{Q}{r} \text {. }
$$

The electromagnetic tensor $F_{\alpha \beta}=A_{\beta, \alpha}-A_{\alpha, \beta}$ has the only one nonzero component

$$
F_{t r}=-F_{r t}=-\frac{Q}{r^{2}} .
$$

Motion of a charged particle of mass $m$ and charge $q$ in the combined background of gravitational and electric fields is governed by the Lorentz equation. Symmetries of 
the combined background imply two integrals of motion that correspond to temporal and spatial components of the canonical four-momentum of the charged particle:

$$
\begin{aligned}
\frac{P_{t}}{m} & =-\mathcal{E} \equiv-\frac{E}{m}=u_{t}-\frac{q Q}{m r}, \\
\frac{P_{\phi}}{m} & =\mathcal{L} \equiv \frac{L}{m}=u_{\phi},
\end{aligned}
$$

where $\mathcal{E}$ and $\mathcal{L}$ denote the specific energy and the specific angular momentum of the charged particle, respectively. The motion is concentrated in the central planes, and we can choose for simplicity the equatorial plane $(\theta=\pi / 2)$. The three non-vanishing components of the equation of motion (45) take the form

$$
\begin{aligned}
\frac{\mathrm{d} u^{t}}{\mathrm{~d} \tau} & =\frac{u^{r}[Q r-2 M(e r+Q)]}{r(r-2 M)^{2}} \\
\frac{\mathrm{d} u^{r}}{\mathrm{~d} \tau} & =\frac{e Q}{r^{2}}+\frac{\mathcal{L}^{2}(r-2 M)}{r^{4}}-\frac{M\left[e^{2}-\left(u^{r}\right)^{2}\right]}{r(r-2 M)}, \\
\frac{\mathrm{d} u^{\phi}}{\mathrm{d} \tau} & =-\frac{2 \mathcal{L} u^{r}}{r^{3}}, \\
\text { where } & e=\mathcal{E}-\frac{q Q}{m r} .
\end{aligned}
$$

The normalization condition for a massive particle $u^{\mu} u_{\mu}=-1$ implies the existence of the effective potential governing the radial motion of the charged particles

$$
V_{\text {eff }}(r)=\frac{\mathcal{Q}}{r}+\sqrt{f(r)\left(1+\frac{\mathcal{L}^{2}}{r^{2}}\right)},
$$

where $\mathcal{Q}=Q q / m$ is a parameter characterizing the electric interaction between the charges of the particle and the black hole. Without loss of generality we set the mass of the black hole to be $M=1$, expressing thus all quantities in units of $M$.

The local extrema of the effective potential $V_{\text {eff }}$ govern the circular orbits by the relation [91]

$$
r^{2}(J-1)+\mathcal{L}^{2}(r-3)=0,
$$

where

$$
J=\frac{\mathcal{Q}}{r} \sqrt{\frac{(r-2)\left(\mathcal{L}^{2}+r^{2}\right)}{r}} .
$$

The radial profiles of the specific angular momentum of the circular orbits are given by relations governing two families of these orbits

$$
\mathcal{L}_{ \pm}^{2}=\frac{r}{(r-3)^{2}}\left[-\mathcal{Q}^{2}-3 r+\frac{\mathcal{Q}^{2} r}{2}+r^{2} \pm \mathcal{Q} \sqrt{\mathcal{Q}^{2}-12 r+4 r^{2}}\left(1-\frac{r}{2}\right)\right],
$$

The limits on the angular velocity of the circular orbits as measured by distant static observers $\Omega=d \phi / d t$ are again given by the angular velocities related to the photon motion $\Omega_{ \pm}$. The possible values of $\Omega$ are thus restricted by

$$
\Omega_{-} \leq \Omega \leq \Omega_{+}, \quad \Omega_{ \pm}= \pm \frac{\sqrt{f(r)}}{r} .
$$

The limiting values of $\Omega$ can be again applied in estimates of the efficiency of the electric Penrose process. 


\subsection{Energy of Ionized Particles}

Assume the decay of particle 1 into two fragments 2 and 3 close to the event horizon of a weakly charged Schwarzschild black hole. We can give the following conservation laws for situations before and after decay-assuming motion in the equatorial plane, they take the form

$$
\begin{array}{r}
E_{1}=E_{2}+E_{3}, \quad L_{1}=L_{2}+L_{3}, \quad q_{1}=q_{2}+q_{3}, \\
m_{1} \dot{r_{1}}=m_{2} \dot{r_{2}}+m_{3} \dot{r_{3}}, \quad m_{1} \geqslant m_{2}+m_{3},
\end{array}
$$

where a dot indicates derivatives with respect to the particle proper time $\tau$. The abovepresented conservation laws imply relation

$$
m_{1} u_{1}^{\phi}=m_{2} u_{2}^{\phi}+m_{3} u_{3}^{\phi} .
$$

Using relations $u^{\phi}=\Omega u^{t}=\Omega e / f(r)$, where $e_{i}=\left(E_{i}+q_{i} A_{t}\right) / m_{i}$, with $i=1,2,3$ indicating the particle number, the equation (123) can be modified to the form

$$
\Omega_{1} m_{1} e_{1}=\Omega_{2} m_{2} e_{2}+\Omega_{3} m_{3} e_{3}
$$

enabling to express the third particle energy $E_{3}$ in the form

$$
E_{3}=\frac{\Omega_{1}-\Omega_{2}}{\Omega_{3}-\Omega_{2}}\left(E_{1}+q_{1} A_{t}\right)-q_{3} A_{t},
$$

where $\Omega_{i}=d \phi_{i} / d t$ is an angular velocity of $i$ th particle.

To maximize the third, particle energy we chose again an electrically neutral first particle, $q_{1}=0$. We also chose $E_{1}=m_{1}$ or $\mathcal{E}=1$. In this case, the angular velocity for the first particle 1 has the following simple form

$$
\Omega_{1}=\frac{1}{r^{2}} \sqrt{2(r-2)} .
$$

The energy of the ionized third particle is maximal, if $\left(\Omega_{1}-\Omega_{2}\right) /\left(\Omega_{3}-\Omega_{2}\right)$ is maximized. This can be done when the angular momentum of the fragments takes their limiting values, implying the relation

$$
\left.\frac{\Omega_{1}-\Omega_{2}}{\Omega_{3}-\Omega_{2}}\right|_{\max }=\frac{1}{\sqrt{2 r_{\text {ion }}}}+\frac{1}{2},
$$

with $r_{\text {ion }}$ being the ionization radius. The ratio (127) decreases with increasing $r_{\text {ion }}$ being maximal while $r_{\text {ion }}$ is approaching the event horizon. Thus, at $r_{\text {ion }}=2$, the ratio (127) is equal to unity, and the expression for the energy of the ionized third particle takes the form [91]

$$
E_{3}=\left(\frac{1}{\sqrt{2 r_{\text {ion }}}}+\frac{1}{2}\right) E_{1}+\frac{q_{3} Q}{r_{\text {ion }}} .
$$

The charged particle is accelerated by the Coulombic repulsive force acting between the black hole and particle, while $q_{3}$ and $Q$ have the same sign. We defined the ratio between the energies of ionized and neutral particles representing the efficiency of the acceleration process. Using the standard units in expressing the black hole mass and characterizing the third particle by $q_{3}=Z e$ and the first particle by $m_{1} \approx A m_{n}$, where $Z$ and $A$ are the atomic and mass numbers, $e$ is the elementary (proton) charge and $m_{n}$ is the nucleon mass, the efficiency of the electric Penrose process can be given as [91]

$$
\eta_{E P P}=\frac{E_{3}}{E_{1}}=\frac{1}{2}+\sqrt{\frac{G M}{2 c^{2} r_{\text {ion }}}}+\frac{Z e Q}{A m_{n} c^{2} r_{\text {ion }}} .
$$


For the ionization point approaching the event horizon, $r_{\text {ion }} \approx 2 G M / c^{2}$, the condition $E_{3}>E_{1}$ is satisfied for arbitrary positive values of the black hole charge, $Q>0$. For the ionization (splitting) point approaching the ISCO radius, i.e., $r_{\text {ion }}=6 G M / c^{2}$, the condition $E_{3}>E_{1}$ is satisfied for the black hole charge satisfying the relation

$$
Q \gtrsim 5.8 \times 10^{11} \frac{A}{Z} \frac{M}{M_{\odot}} \mathrm{Fr},
$$

slightly greater than the lower limit of the estimated realistic limits of the black hole charge given by (105).

The maximal energy of ionized particle accelerated by the non-rotating weakly charged black holes can be determined from (129)_by using the uppermost realistic limit of the charge (105) we arrive to

$$
E_{\mathrm{ion}}^{\max } \approx 1.01 \times 10^{6} \mathrm{Z} \frac{Q}{10^{18} \mathrm{Fr}} \frac{M_{\odot}}{M} \mathrm{GeV},
$$

or, equivalently, $\approx 1620 \mathrm{erg}$. The ratio of energies of ionized and neutral particles is then equal to $E_{\mathrm{ion}}^{\max } / E_{n} \approx 10^{6}$. In sharp contrast to the magnetic Penrose process [14,28], where the energy of ionized particle increases with increasing black hole mass, for non-rotating weakly charged black holes, the energy of a charged particle is inversely proportional to the black hole mass. The maximal energy is determined by the limiting value of the black hole charge-to-mass ratio $Q / M$ (see the limits (105) and the charge of the ionized particle $\mathrm{Ze}$. Therefore, the maximal energy of ionized particle accelerated by the weakly charged non-rotating black hole is similar for both stellar mass and supermassive black holes in clear contrast to the MPP.

It follows from the presented results that even the electric Penrose process can lead to acceleration of protons to the ultra-high energies and could be an explanation of the UHECRs - increasing the black hole charge for a given black hole mass, one can reach the UHECR orders of energies. A central charge of the black hole, in this case, is still smaller than the maximal theoretical charge limit by many orders of magnitude.

The constraint on mass and charge of the black hole in Sgr A* allow the black hole to act as a PeVatron of charged particles, with energy of the accelerated protons being of the order of $10^{15} \mathrm{eV}$, similarly to the case of the MPP. The electric Penrose process could be thus considered as an alternative explanation of the cosmic ray knee when applied to the Galactic supermassive centre black hole Sgr A* [91].

\section{Radiative Penrose Process}

Finally, we discuss the newly discovered variant of the Penrose process that is related to the radiative self-force connected with the synchrotron radiation of charged particles moving in the ergosphere of a magnetized Kerr black hole. Here, we present a wide range of possible variants of the particle motion undergoing the radiative Penrose process (RPP), representing a gain of rotational energy of the black hole by a single radiating particle, and a comparison of the properties of the motion around the Kerr black holes and Kerr naked singularities. The origin of the Penrose process is in the RPP case connected with the special class of radiated photons that have negative energy relative to distant observers [47,92].

\subsection{Landau-Lifshitz Equations of Motion under Radiative Force}

Charged particle motion in curved spacetimes under influence of the external electromagnetic force combined with the radiation reaction self-force is determined by the DeWitt-Brehme equation [78]. However, the DeWitt-Brehme equation contains the thirdorder time derivative of coordinates giving pre-accelerating solutions when no external forces exist. Fortunately, the equations of motion can be modified by using derivatives of 
the external forces instead of the third-order term in the Landau-Lifshitz method in its covariant form [77], leading to the equations

$$
\frac{D u^{\mu}}{d \tau}=\frac{q}{m} F^{\mu} u^{v}+\frac{2 q^{2}}{3 m}\left[F_{\beta ; \mu}^{\alpha} u^{\beta} u^{\mu}+\frac{q}{m}\left(F^{\alpha}{ }_{\beta} F^{\beta}{ }_{\mu}+F_{\mu v} F^{v}{ }_{\sigma} u^{\sigma} u^{\alpha}\right) u^{\mu}\right],
$$

with the covariant coordinate derivative denoted by semicolon. The Landau-Lifshitz equations given by the relations (90) are second-order differential equations fulfilling the principle of inertia so that the runaway solutions are not allowed [77]. In the background of the magnetized Kerr black holes, Equation (90) is very complicated and long even for the equatorial motion-for this reason we dis not present here its explicit form.

During the study of the consequences of the calculations of the particle motion determined by the Landau-Lifshitz equations, when usually the radiative forces imply decreasing of the particle energy, an unexpected effect of energy gain of the radiating particle has been demonstrated for the motion inside the ergosphere of magnetized Kerr black holes. This RPP was observed solely in the ergosphere of the Kerr black hole; the effective ergosphere related to the moving charged particles plays absolutely no role in this phenomenon.

We present first a standard situation of energy damping due to the radiative force, acting outside the ergosphere, in Figure 7, comparing motion under similar conditions in the field of Kerr black holes and Kerr naked singularities, demonstrating only quantitative differences for these different kinds of the Kerr spacetime.

\subsection{Negative Energy Photons inside the Ergosphere and Energy Gain by Radiating Particle}

In the ergosphere, any particle (charged or uncharged) has to be co-rotating with the black hole rotation-distant static observers measure $u^{\phi}>0$. On the other hand, the energy of a particle as related to the distant observers can be negative, but the locally measured energy is always positive; the most convenient basic local observers are the ZAMO. A local observer sees the particle in counter-rotating motion, if its $\phi$ component of covariant four-velocity is negative $u_{\phi}<0$. In the ergosphere, radiated photons are attaining negative energies and negative angular momenta $\left(E_{\mathrm{ph}}<0, L_{\mathrm{ph}}<0\right)$ related to distant observers, if the radiating particle satisfies the following conditions

$$
u_{t}>0, \quad u_{\phi}<0 .
$$

The photons emitted by the relativistic charged particles attain negative energy only if emitted (locally) backwards with respect to the $\mathrm{BH}$ rotation and radiating particle must be locally counter-rotating with $u_{\phi}<0$.

Photons with negative energy can exist only inside the ergosphere and have to be captured by the black hole finally [92]. Being emitted by a radiating charged particle with properly chosen energy and angular momentum, they cause the spin down of the black hole due to the extraction of its rotation energy—such photons are at the superradiance modes of the electromagnetic wave equations.

The negative energy emission has to be enforced by the relativistic beaming effect. We can demonstrate the basic character of the RPP demonstrating the role of the beaming looking at the situation from the point of view of LNRF-see Figure 8. We assumed a source radiating isotropically in the LNRF; then, the part of the radiated photons with negative energy, directed with counter-rotating orientation related to distant observers, is smaller than those of the positive energy (corotating). However, if the source is moving relatively to LNRF, the relativistic beaming causes amplification of the radiation (energy) in direction of the motion, so for a source counter-rotating relative to the LNRF, the negative energy photons are amplified and the total radiated energy can be negative. 

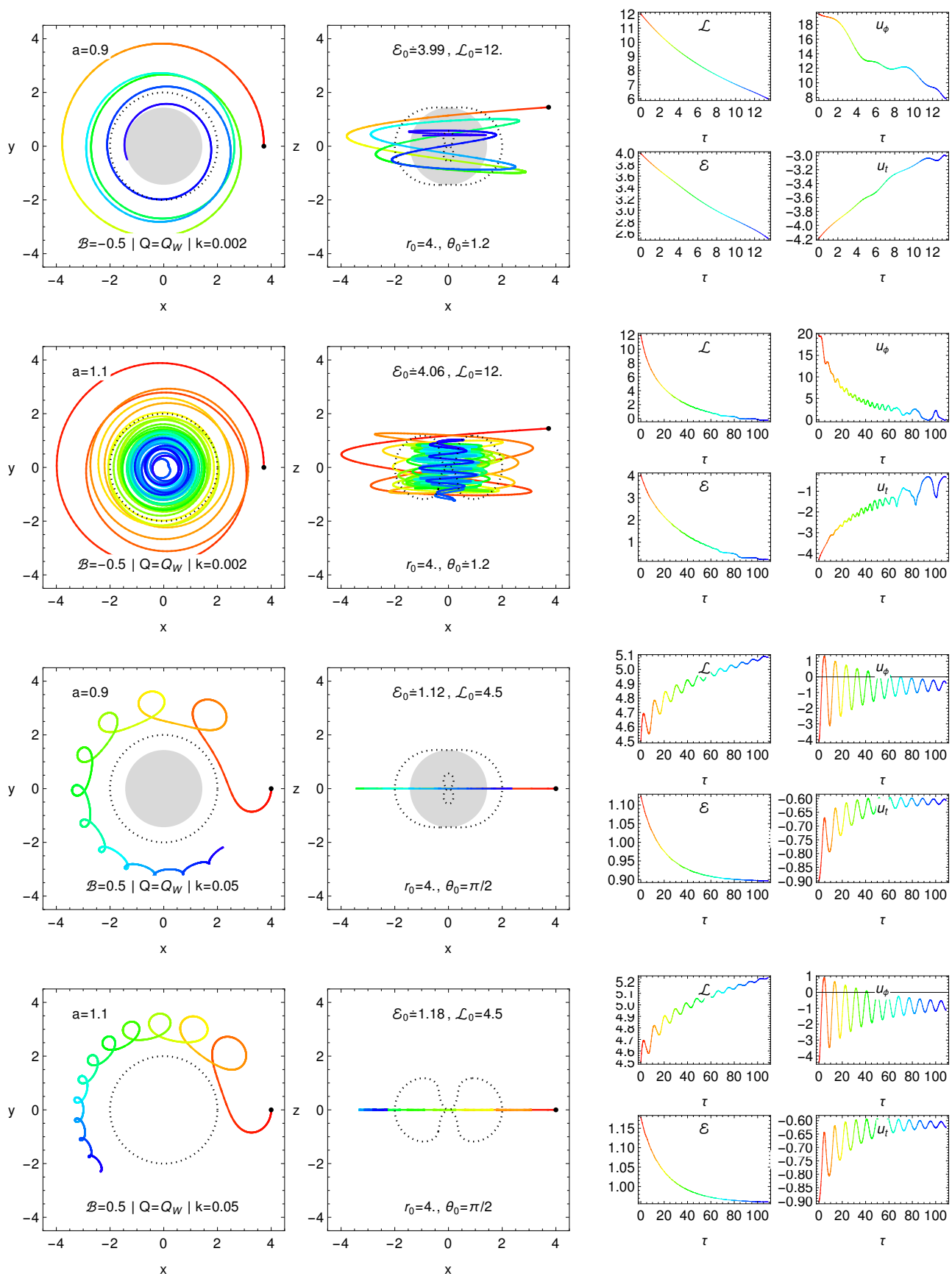

Figure 7. Charged particle radiation outside black hole ergosphere. Radiating charged particle trajectories demonstrate the role of the self-reaction force for two different kinds of orbits, comparing them for the black hole and naked singularity spacetimes. We show projections of particle trajectory into $x y, x z$ planes, particle specific energy $\mathcal{E}$, radial coordinate $r$, and covariant component $u_{t}, u_{\phi}$, as functions of proper time $\tau$. Change in the colors denotes the evolution with the proper time, gray disk denotes $\mathrm{BH}$ interior, and dotted curve shows the boundaries of the egrosphere. 

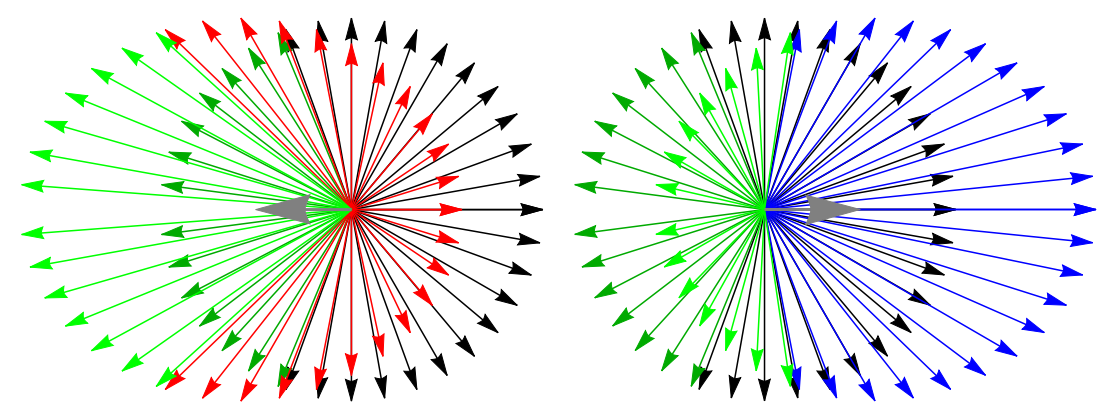

Figure 8. Distribution of the photons with negative and positive energy in the LNRF located in the ergosphere of a Kerr black hole, and the effect of beaming due to the motion in the sense of the black hole rotation (right) and in opposite direction (left), giving an illustrative explanation of the RPP.

Now we concentrate on the dynamics of charged particles moving under the influence of the radiation reaction self-force by emitting synchrotron photons in the ergosphere, gaining in this way the rotational energy of the black hole, if the negative-energy photons prevail in the radiation.

We demonstrate the energy gain effect of the RPP integrating the Landau-Lifshitz Equation (90) inside the ergosphere of a magnetized Kerr metric immersed in the uniform (Wald) magnetic field. We introduce the following independent parameters

$$
\mathcal{E}=\frac{E}{m}, \quad \mathcal{L}=\frac{L}{m}, \quad \mathcal{B}=\frac{q B M}{2 m}, \quad k=\frac{2}{3} \frac{q^{2}}{m M} ;
$$

the magnetic parameter $\mathcal{B}$ gives the ratio of the Lorentz force to the gravitational force, $\mathcal{E}$ and $\mathcal{L}$ are the specific energy and specific axial angular momentum of the charged particle, respectively, and $k$ is the radiation parameter reflecting the energy evolution. Results of the numerical integration of Equation (90) are demonstrated in Figs with initial conditions shown inside the plots.

We demonstrate the basic three situations related to the RPP in Figure 9. Namely, we demonstrate orbit with increasing energy in the ergosphere, and particle escaping to infinity loosing energy outside the ergosphere; the "floating orbit" crossing repeatedly the ergosphere while gaining energy inside and loosing energy outside the ergosphere; and the orbits with increasing energy but finishing in the black hole.

We demonstrate a comparison of the escaping orbits gaining energy in the ergosphere of a Kerr black hole and naked singularity in Figure 10. The energy gain at the edge of the ergosphere can represent typically one order, being nearly the same in both black holes and naked singularities.

In Figure 11, we compare the orbits of radiating particles in the Kerr black hole background for the cases with the Wald charge and with vanishing of the Wald charge, demonstrating higher efficiency for escaping of the particle in the background with the Wald charge. 

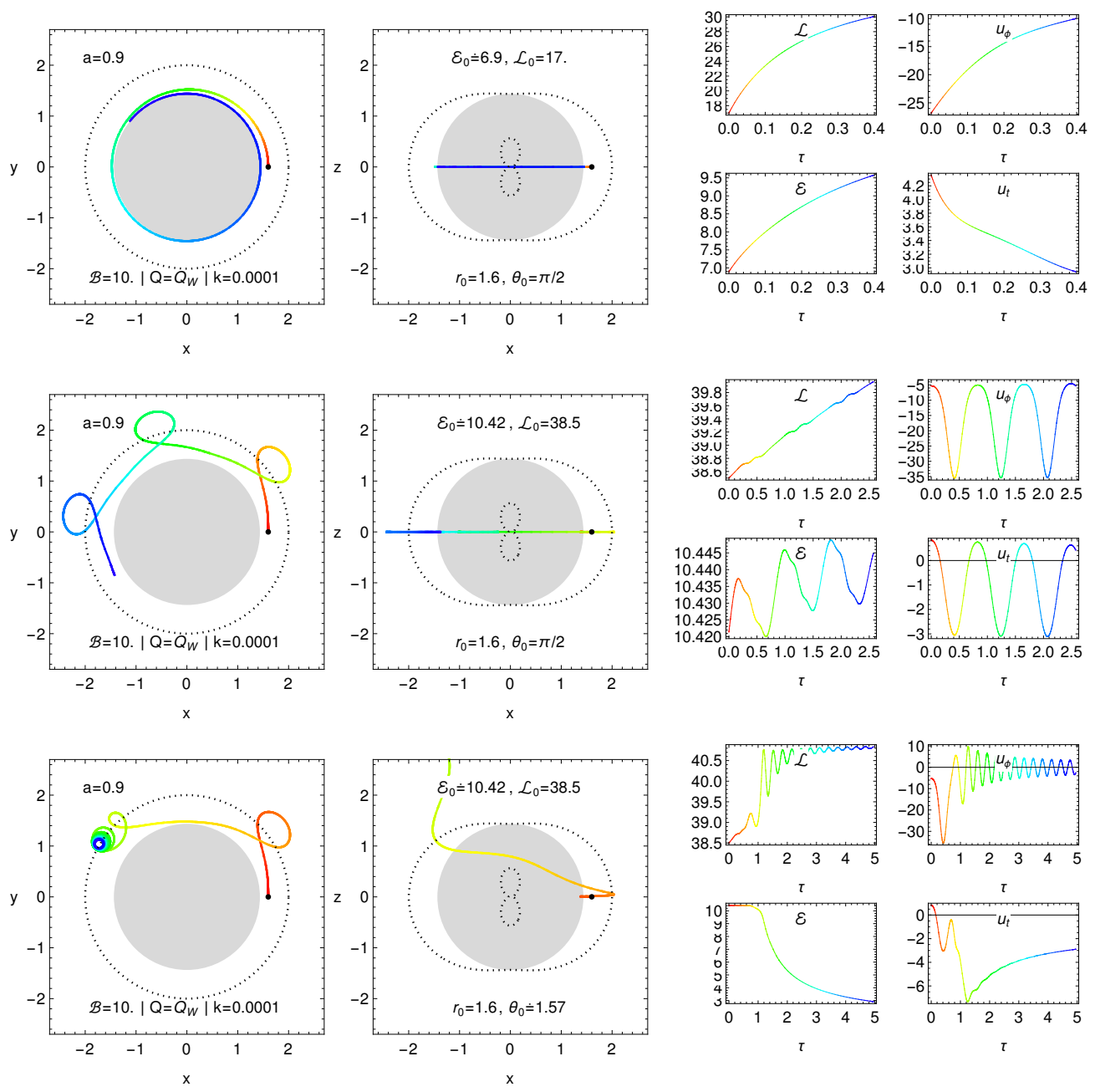

Figure 9. The energy gain in the RPP due to radiating force in the ergosphere. Three basic types of the orbits are presented: the collapsing orbit gaining the energy while being finished in the black hole, the floating orbit where the radiating particle successively gains energy during the motion inside the ergosphere and looses the energy during the motion outside the ergosphere, and the escaping orbit where the particle is gaining energy in the ergosphere and loosing the energy outside.

The magnitude of the energy gain in obtained in the RPP depends both on the parameters governing the electromagnetic forces $\mathcal{B}$ and $k$ and on the length and direction of the trajectory of the charged particle inside the ergosphere. Nevertheless, we observed strongly "chaotic" behavior of the properties of the radiative Penrose process due to the chaotic origin of the motion - the trajectory length and gained energy can significantly differ for trajectories with different initial conditions. Charged particles starting their motion inside the ergosphere with a large pitch angle with respect to the equatorial plane (i.e., having a significant $\theta$ component of the four-momentum) have a short trajectory inside the ergosphere as such particles have a tendency to escape soon in the vertical direction along the magnetic field lines. For particles with a trajectory lying in or very close to the equatorial plane, their motion in the ergosphere is much longer, allowing significantly larger period of energy gaining. Note that, in general, the motion of ultra-relativistic particles demonstrates an instability inside the ergosphere, and a small change in $p_{\theta}$ (e.g., due to influence of other particle or photon) may cause the particle to fall into the black hole or its escape in the vertical direction along the magnetic field lines [14,28]. 

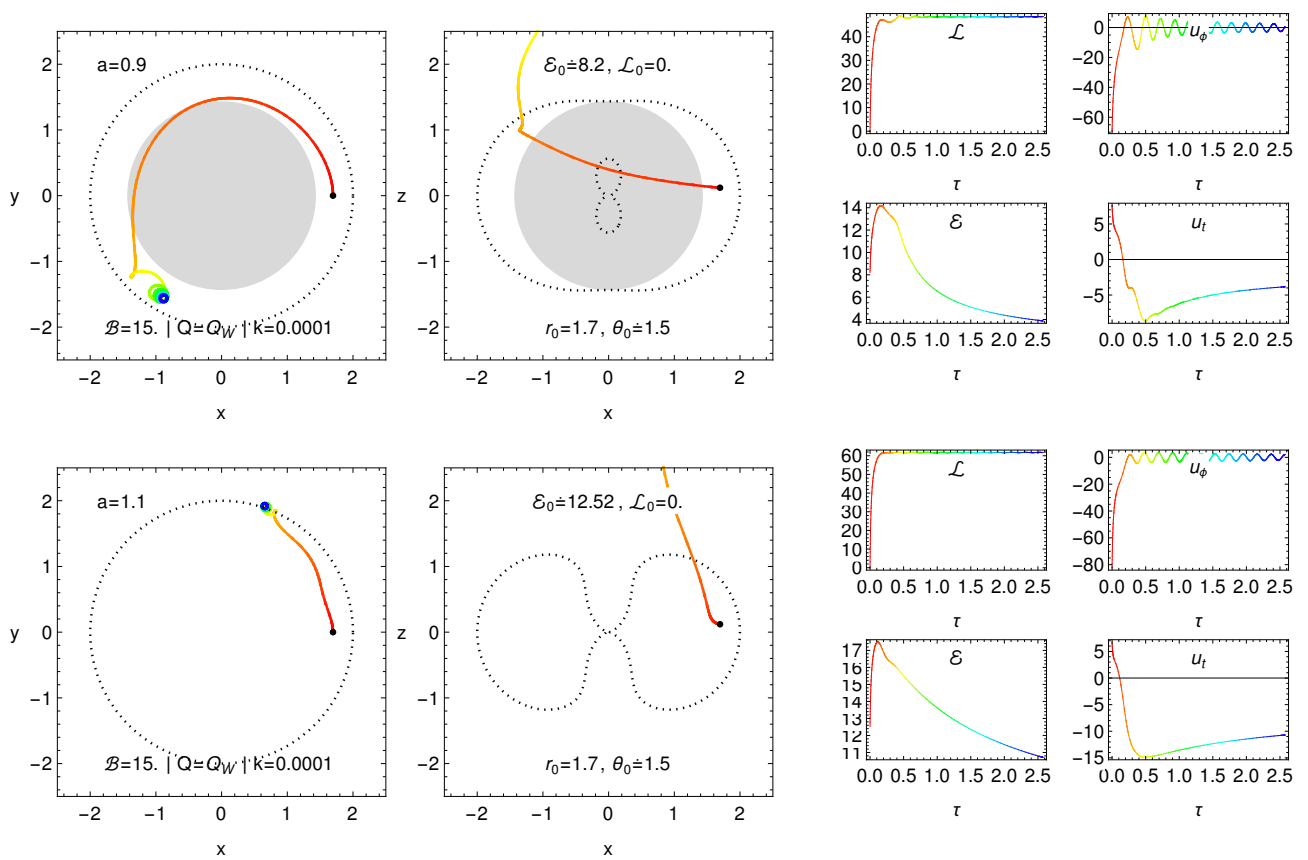

Figure 10. Charged particle escape after gaining energy due to the RPP. We compared the character of the orbits in the black hole and naked singularity spacetimes.
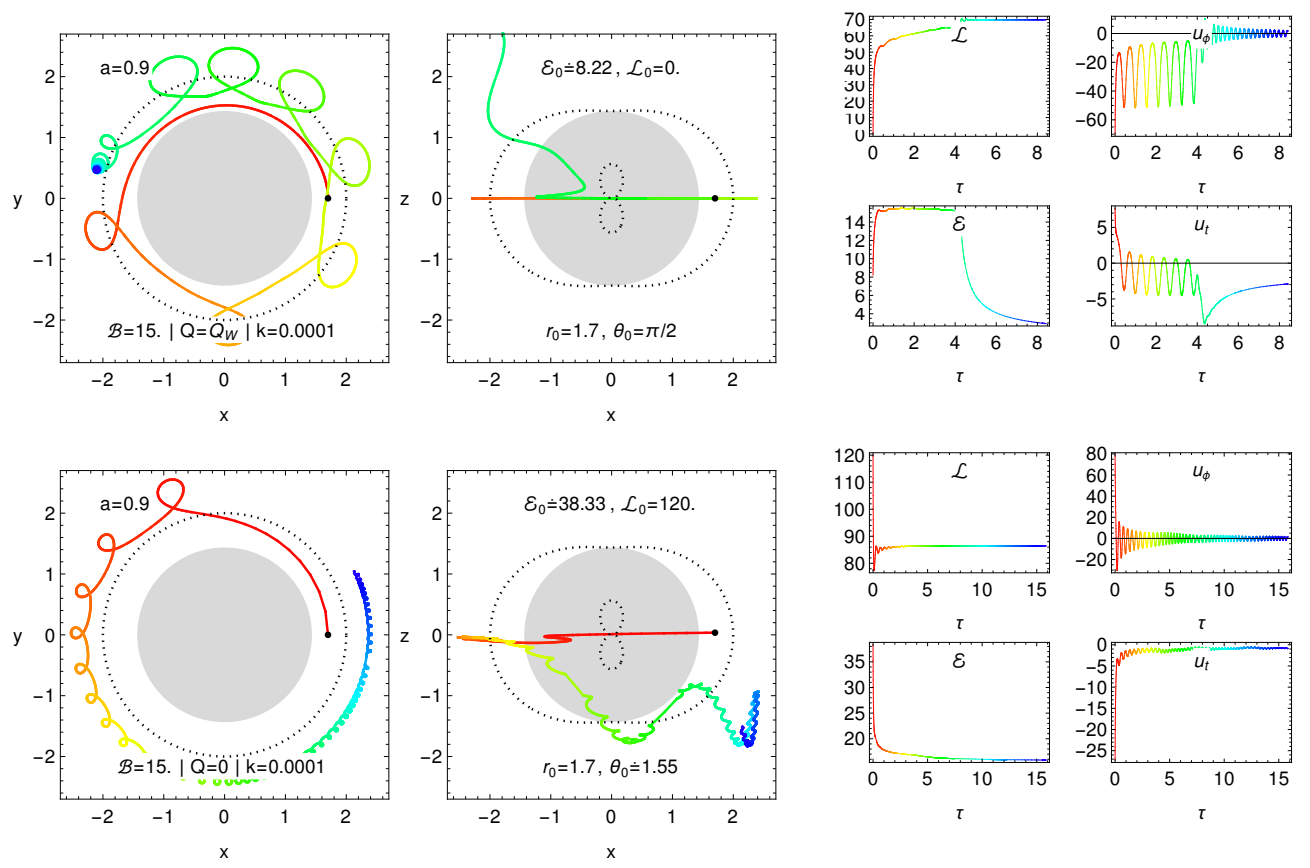

Figure 11. Comparison of the charged particle orbits in and outside ergosphere in the field of Kerr black holes having the Wald charge and without the charge.

Outside the ergosphere, the energy of emitted photons with respect to a distant observer is always positive (there are no photons with negative energy outside the ergosphere [92]) causing a decrease in the particle energy, and the charged particle continues its motion in the magnetic field loosing its energy in the standard manner [30]. Usually, the charged particles loose a significant amount of their energy gained in the ergosphere just after they leave the ergosphere-this fact implies the possibility of significant signatures in the astrophysical phenomena connected with the innermost parts of accretion structures observed in active galactic nuclei. Notice, however, the possibility of slow decrease of the particle energy observed in the case of the Kerr naked singularity-a detailed undergoing 
study is necessary to clear up if it could be an effect of the spacetime origin or if it is related only to the specific choice of the initial conditions for the motion of the radiating particles. Generally, we can say [47] that the radiation is maximal for the direction of the motion perpendicular to the magnetic field lines and minimal (basically zero) for the motion along the magnetic field lines, i.e., along the axis of the black hole rotation where the ultra-high energy particles are expected.

\section{Conclusions}

The combined influence of rotating black holes and external magnetic fields can be demonstrated by important phenomena related to accretion disks or tori where extraction of energy into the jets could be related to the MPP representing their significant role in astrophysics, especially in the generation of UHECR [28]. It is quite interesting that generation of the UHECR could be obtained in the recently introduced electric Penrose process related to weakly charged non-rotating black holes [91].

The external magnetic fields have the strongest role near the black holes horizon, namely, in the ergosphere or the effective ergosphere, due to the effect of the frame dragging of the spacetime on the magnetic field, enabling the extraction of rotational energy of the black hole.

Assuming the ionized Keplerian disks, and the chaotic scattering of the ionized particles, the MPP can lead to the creation of protons and ions with energy reaching $E=10^{21} \mathrm{eV}$, or even $E=10^{22} \mathrm{eV}$, in the vicinity of the rotating black holes of mass $M \sim 10^{10} M_{\odot} \mathrm{im}$ mersed in the external uniform magnetic field of strength $B \sim 10^{5} \mathrm{G}$. For ions accelerated in similar conditions by the same black hole, the energy is decreased by the factor given by the ratio of the specific charge of protons and the ion. For protons and ions, the energy loss due to the synchrotron radiation and related back-reaction on their motion can be negligible near such supermassive black holes, but it is strongly significant for electrons, giving a clear explanation of the fact that no highly energetic electrons are observed in UHECR. The strong back-reaction on the moving particles is the reason why we cannot observe so highly energetic particles (electron, proton, ions) coming from magnetars, where the efficiency of the acceleration is comparable to those of the supermassive black holes.

The maximal proton energy that can be obtained near the SgrA* black hole, where $M \sim 4 \times 10^{6} M_{\odot}$ and $B \sim 10^{2} \mathrm{G}$, takes the value $E \sim 10^{16} \mathrm{eV}$ corresponding to the knee of the observational data of registered UHECRs [28].

Surprisingly, efficient acceleration of protons and ions could be relevant also for non-rotating Schwarzschild black holes if they carry an astrophysically acceptable (small) electric charge; the efficiency of such an electric Penrose process is inversely dependent on the black hole mass, and can work isotropically in contract with the MPP [91].

Even more interesting is another recently discovered variant-the radiative Penrose process that could occur due to the synchrotron radiation because of radiating negative energy photons [92] and the related back-reaction on the radiating particle in the ergosphere of Kerr black holes-efficient acceleration by one order of energy is possible, along with the case of floating orbits that successively extract and radiate the rotational energy while realizing an epicyclic motion [47]. Clearly, the radiative Penrose process can have relevant consequences for the creation of jets, and their appearance, being thus inspiration for more detailed magneto-hydrodynamic studies of tori and related jets.

For the motion in the strong magnetic field near the black hole, the radiation backreaction is negligible for protons and ions, so they are leaving the acceleration region with the energy ratio given by the ratio of their specific charges. However, the radiation back-reaction is important during travel from the source to the Earth in weak intergalactic fields, where it differs for protons and ions. Therefore, the ratio of the energies of protons and ions observed simultaneously at Earth can give significant information on the distance of the source of the UHECRs. The models of UHECR acceleration by the magnetic Penrose process could thus be relevant for the search for UHECR sources, giving thus a special new application of the magnetic Penrose process, increasing its role in astrophysics. 
Author Contributions: Conceptualization, Z.S.; methodology, A.T.; writing-original draft preparation, Z.S. and A.T.; writing-editing, M.K.; visualization, M.K.; supervision, Z.S. All authors have read and agreed to the published version of the manuscript.

Funding: The authors would like to acknowledge the Research Centre for Theoretical Physics and Astrophysics and Institute of Physics of Silesian University in Opava for institutional support. Z. S. acknowledges the support of the Grant No. 19-03950S of Czech Science Foundation (GACR).

Acknowledgments: The authors would like to acknowledge the Research Centre for Theoretical Physics and Astrophysics and Institute of Physics of Silesian University in Opava for institutional support. Z. S. acknowledges the support of the Grant No. 19-03950S of the Czech Science Foundation (GAČR).

Conflicts of Interest: The authors declare no conflict of interest.

\section{References}

1. Penrose, R. Gravitational Collapse: the Role of General Relativity. Nuovo C. Riv. Ser. 1969, 1, 252.

2. Tchekhovskoy, A. Launching of Active Galactic Nuclei Jets. The Formation and Disruption of Black Hole Jets. In Astrophysics and Space Science Library; Contopoulos, I., Gabuzda, D., Kylafis, N., Eds.; Springer International Publishing Switzerland: Cham, Switzerland, 2015; Volume 414, p. 45. [CrossRef]

3. Blandford, R.D.; Znajek, R.L. Electromagnetic extraction of energy from Kerr black holes. Mon. Not. R. Astron. Soc. 1977, 179, 433-456. [CrossRef]

4. Dadhich, N.; Tursunov, A.; Ahmedov, B.; Stuchlík, Z. The distinguishing signature of magnetic Penrose process. Mon. Not. R. Astron. Soc. 2018, 478, L89-L94. [CrossRef]

5. Tursunov, A.; Dadhich, N. Fifty Years of Energy Extraction from Rotating Black Hole: Revisiting Magnetic Penrose Process. Universe 2019, 5, 125. [CrossRef]

6. Stuchlík, Z.; Kološ, M.; Tursunov, A.A. Magnetized Black Holes: Ionized Keplerian Disks and Acceleration of Ultra-High Energy Particles. Proceedings 2019, 17, 13. [CrossRef]

7. Abramowicz, M.A.; Fragile, P.C. Foundations of Black Hole Accretion Disk Theory. Living Reviews in Relativity 2013, 16, 1. [CrossRef]

8. Novikov, I.D.; Thorne, K.S. Astrophysics of black holes. In Black Holes (Les Astres Occlus); Dewitt, C., Dewitt, B.S., Eds.; Gordon \& Breach: New York, NY, USA, 1973; pp. 343-450.

9. Kozlowski, M.; Jaroszynski, M.; Abramowicz, M.A. The analytic theory of fluid disks orbiting the Kerr black hole. Astron. Astrophys. 1978, 63, 209-220.

10. Pugliese, D.; Stuchlík, Z. Ringed Accretion Disks: Equilibrium Configurations. Astrophys. J. Suppl. 2015, 221, 25. [CrossRef]

11. Pugliese, D.; Stuchlík, Z. Ringed Accretion Disks: Instabilities. Astrophys. J. Suppl. 2016, 223, 27. [CrossRef]

12. Pugliese, D.; Stuchlík, Z. Ringed Accretion Disks: Evolution of Double Toroidal Configurations. Astrophys. J. Suppl. 2017, 229, 40. [CrossRef]

13. Stuchlík, Z. Influence of the RELICT Cosmological Constant on Accretion Discs. Mod. Phys. Lett. 2005, 20, 561-575. [CrossRef]

14. Stuchlík, Z.; Kološ, M.; Kovář, J.; Slaný, P.; Tursunov, A. Influence of Cosmic Repulsion and Magnetic Fields on Accretion Disks Rotating around Kerr Black Holes. Universe 2020, 6, 26. [CrossRef]

15. Stuchlik, Z. The Motion of Test Particles in Black-Hole Backgrounds with Non-Zero Cosmological Constant. Bull. Astron. Institutes Czechoslov. 1983, 34, 129.

16. Stuchlík, Z.; Hledík, S. Some properties of the Schwarzschild-de Sitter and Schwarzschild-anti-de Sitter spacetimes. Phys. Rev. D 1999, 60, 044006. [CrossRef]

17. Stuchlík, Z.; Hledík, S.; Novotný, J. General relativistic polytropes with a repulsive cosmological constant. Phys. Rev. D 2016, 94, 103513. [CrossRef]

18. Misner, C.W.; Thorne, K.S.; Wheeler, J.A. Gravitation; Princeton University Press: Princeton, NJ, USA, 1973.

19. de Felice, F. Repulsive Phenomena and Energy Emission in the Field of a Naked Singularity. Astron. Astrophys. $1974,34,15$.

20. Stuchlík, Z. Equatorial circular orbits and the motion of the shell of dust in the field of a rotating naked singularity. Bull. Astron. Institutes Czechoslov. 1980, 31, 129-144.

21. Stuchlík, Z.; Hledík, S.; Truparová, K. Evolution of Kerr superspinars due to accretion counterrotating thin discs. Class. Quantum Gravity 2011, 28, 155017. [CrossRef]

22. Gimon, E.G.; Horava, P. Over-Rotating Black Holes, Godel Holography and the Hypertube. arXiv 2004, arXiv:hep-th/hepth/0405019.

23. Stuchlík, Z.; Schee, J. Appearance of Keplerian discs orbiting Kerr superspinars. Class. Quantum Gravity $2010,27,215017$. [CrossRef]

24. Stuchlík, Z.; Schee, J. Observational phenomena related to primordial Kerr superspinars. Class. Quantum Gravity 2012, $29,065002$. [CrossRef] 
25. Stuchlík, Z.; Schee, J. Ultra-high-energy collisions in the superspinning Kerr geometry. Class. Quantum Gravity 2013, $30,075012$. [CrossRef]

26. Kološ, M.; Tursunov, A.; Stuchlík, Z. Possible signature of the magnetic fields related to quasi-periodic oscillations observed in microquasars. Eur. Phys. J. 2017, 77, 860. [CrossRef]

27. Stuchlík, Z.; Kološ, M. Acceleration of the charged particles due to chaotic scattering in the combined black hole gravitational field and asymptotically uniform magnetic field. Eur. Phys. J. 2016, 76, 32. [CrossRef]

28. Tursunov, A.; Stuchlík, Z.; Kološ, M.; Dadhich, N.; Ahmedov, B. Supermassive Black Holes as Possible Sources of Ultrahigh-energy Cosmic Rays. Astrophys. J. 2020, 895, 14. [CrossRef]

29. Wald, R.M. Black hole in a uniform magnetic field. Phys. Rev. D 1974, 10, 1680-1685. [CrossRef]

30. Tursunov, A.; Kološ, M.; Stuchlík, Z.; Galtsov, D.V. Radiation Reaction of Charged Particles Orbiting a Magnetized Schwarzschild Black Hole. Astrophys. J. 2018, 861, 2. [CrossRef]

31. Tursunov, A.A.; Kološ, M.; Stuchlík, Z. Orbital widening due to radiation reaction around a magnetized black hole. Astron. Nachrichten 2018, 339, 341-346. [CrossRef]

32. Ruffini, R. On the energetics of black holes. In Black Holes (Les Astres Occlus); Dewitt, C., Dewitt, B.S., Eds.; Gordon \& Breach: New York, NY, USA, 1973; pp. 451-546.

33. Komar, A. Positive-Definite Energy Density and Global Consequences for General Relativity. Phys. Rev. 1963, 129, 1873-1876. [CrossRef]

34. Carter, B. Black hole equilibrium states. In Black Holes (Les Astres Occlus); Dewitt, C., Dewitt, B.S., Eds.; Gordon \& Breach: New York, NY, USA, 1973; pp. 57-214.

35. Christodoulou, D. Reversible and Irreversible Transformations in Black-Hole Physics. Phys. Rev. Lett. 1970, $25,1596-1597$. [CrossRef]

36. Christodoulou, D.; Ruffini, R. Reversible Transformations of a Charged Black Hole. Phys. Rev. D 1971, 4, 3552-3555. [CrossRef]

37. Bardeen, J.M.; Press, W.H.; Teukolsky, S.A. Rotating Black Holes: Locally Nonrotating Frames, Energy Extraction, and Scalar Synchrotron Radiation. Astrophys. J. 1972, 178, 347-370. [CrossRef]

38. Ruffini, R.; Wilson, J.R. Relativistic magnetohydrodynamical effects of plasma accreting into a black hole. Phys. Rev. D 1975, 12, 2959-2962. [CrossRef]

39. Bicak, J.; Stuchlik, Z. The fall of the shell of dust on to a rotating black hole. Mon. Not. R. Astron. Soc. 1976, 175, 381-393. [CrossRef]

40. Bicak, J.; Stuchlik, Z. On the latitudinal and radial motion in the field of a rotating black hole. Bull. Astron. Institutes Czechoslov. 1976, 27, 129-133.

41. Bardeen, J.M. Timelike and null geodesics in the Kerr metric. In Black Holes (Les Astres Occlus); Dewitt, C., Dewitt, B.S., Eds.; Gordon \& Breach: New York, NY, USA, 1973; pp. 215-239.

42. Dhurandhar, S.V.; Dadhich, N. Energy-extraction processes from a Kerr black hole immersed in a magnetic field. I. Negativeenergy states. Phys. Rev. D 1984, 29, 2712-2720. [CrossRef]

43. Dhurandhar, S.V.; Dadhich, N. Energy-extraction processes from a Kerr black hole immersed in a magnetic field. II. The formalism. Phys. Rev. D 1984, 30, 1625-1631. [CrossRef]

44. Balbus, S.A.; Hawley, J.F. A powerful local shear instability in weakly magnetized disks. I-Linear analysis. II—Nonlinear evolution. Astrophys. J. 1991, 376, 214-233. [CrossRef]

45. Kološ, M.; Stuchlík, Z.; Tursunov, A. Quasi-harmonic oscillatory motion of charged particles around a Schwarzschild black hole immersed in a uniform magnetic field. Class. Quantum Gravity 2015, 32, 165009. [CrossRef]

46. Tursunov, A.; Stuchlík, Z.; Kološ, M. Circular orbits and related quasi-harmonic oscillatory motion of charged particles around weakly magnetized rotating black holes. Phys. Rev. D 2016, 93, 084012. [CrossRef]

47. Kološ, M.; Tursunov, A.; Stuchlík, Z. Radiative Penrose process: Energy gain by a single radiating charged particle in the ergosphere of rotating black hole. Phys. Rev. D 2021, 103, 024021. [CrossRef]

48. Kovář, J.; Stuchlík, Z.; Karas, V. Off-equatorial orbits in strong gravitational fields near compact objects. Class. Quantum Gravity 2008, 25, 095011. [CrossRef]

49. Kovář, J.; Kopáček, O.; Karas, V.; Stuchlík, Z. Off-equatorial orbits in strong gravitational fields near compact objects - II: halo motion around magnetic compact stars and magnetized black holes. Class. Quantum Gravity 2010, 27, 135006. [CrossRef]

50. Kovář, J. Spiral motion formation in astrophysics. Eur. Phys. J. Plus 2013, 128, 142. [CrossRef]

51. Komissarov, S.S. General relativistic magnetohydrodynamic simulations of monopole magnetospheres of black holes. Mon. Not. R. Astron. Soc. 2004, 350, 1431-1436. [CrossRef]

52. Kovář, J.; Slaný, P.; Stuchlík, Z.; Karas, V.; Cremaschini, C.; Miller, J.C. Role of electric charge in shaping equilibrium configurations of fluid tori encircling black holes. Phys. Rev. D 2011, 84, 084002. [CrossRef]

53. Kovář, J.; Slaný, P.; Cremaschini, C.; Stuchlík, Z.; Karas, V.; Trova, A. Electrically charged matter in rigid rotation around magnetized black hole. Phys. Rev. D 2014, 90, 044029. [CrossRef]

54. Kovář, J.; Slaný, P.; Cremaschini, C.; Stuchlík, Z.; Karas, V.; Trova, A. Charged perfect fluid tori in strong central gravitational and dipolar magnetic fields. Phys. Rev. D 2016, 93, 124055. [CrossRef]

55. Pugliese, D.; Stuchlík, Z. Relating Kerr SMBHs in active galactic nuclei to RADs configurations. Class. Quantum Gravity 2018, 35, 185008. [CrossRef] 
56. Pugliese, D.; Stuchlík, Z. RADs energetics and constraints on emerging tori collisions around super-massive Kerr black holes. Eur. Phys. J. C 2019, 79, 288. [CrossRef]

57. Pugliese, D.; Stuchlík, Z. Jet collision with accreting tori around SMBHs GRHD and light surface constraints in aggregates of misaligned tori. Publ. Astron. Soc. Jpn. 2021. [CrossRef]

58. Cremaschini, C.; Kovář, J.; Slaný, P.; Stuchlík, Z.; Karas, V. Kinetic Theory of Equilibrium Axisymmetric Collisionless Plasmas in Off-equatorial Tori around Compact Objects. Astrophys. J. Suppl. 2013, 209, 15. [CrossRef]

59. Gal'tsov, D.V.; Petukhov, V.I. Black hole in an external magnetic field. Sov. J. Exp. Theor. Phys. 1978, 47, 419.

60. Bicak, J.; Janis, V. Magnetic fluxes across black holes. Mon. Not. R. Astron. Soc. 1985, 212, 899-915. [CrossRef]

61. Moradi, R.; Rueda, J.A.; Ruffini, R.; Wang, Y. The newborn black hole in GRB 191014C proves that it is alive. Astron. Astrophys. 2021, 649, A75. [CrossRef]

62. Zajaček, M.; Tursunov, A.; Eckart, A.; Britzen, S. On the charge of the Galactic centre black hole. Mon. Not. R. Astron. Soc. 2018, 480, 4408-4423. [CrossRef]

63. Bičák, J.; Ledvinka, T. Electromagnetic fields around black holes and Meissner effect. Nuovo C. Ser. 2000, $115,739$.

64. Komissarov, S.S. Blandford-Znajek Mechanism versus Penrose Process. J. Korean Phys. Soc. 2009, 54, 2503. [CrossRef]

65. Punsly, B. Black Hole Gravitohydromagnetics; Springer: Berlin/Heidelberg, Germany, 2001.

66. Frolov, V.P.; Shoom, A.A. Motion of charged particles near a weakly magnetized Schwarzschild black hole. Phys. Rev. D 2010, 82, 084034. [CrossRef]

67. Petterson, J.A. Magnetic field of a current loop around a Schwarzschild black hole. Phys. Rev. D 1974, 10, 3166-3170. [CrossRef]

68. Chandrasekhar, S. The mathematical theory of black holes. In Proceedings of the General Relativity and Gravitation Conference, Padua, Italy, 4-9 July 1984; pp. 5-26.

69. Bičák, J.; Stuchlík, Z.; Balek, V. The motion of charged particles in the field of rotating charged black holes and naked singularities. Bull. Astron. Institutes Czechoslov. 1989, 40, 65-92.

70. Lasota, J.P.; Gourgoulhon, E.; Abramowicz, M.; Tchekhovskoy, A.; Narayan, R. Extracting black-hole rotational energy: The generalized Penrose process. Phys. Rev. D 2014, 89, 024041. [CrossRef]

71. Blandford, R.D.; Payne, D.G. Hydromagnetic flows from accretion disks and the production of radio jets. Mon. Not. R. Astron. Soc. 1982, 199, 883-903. [CrossRef]

72. Greisen, K. End to the Cosmic-Ray Spectrum? Phys. Rev. Lett. 1966, 16, 748-750. [CrossRef]

73. Zatsepin, G.T.; Kuz'min, V.A. Upper Limit of the Spectrum of Cosmic Rays. Sov. J. Exp. Theor. Phys. Lett. 1966, 4, 78.

74. Parsa, M.; Eckart, A.; Shahzamanian, B.; Karas, V.; Zajaček, M.; Zensus, J.A.; Straubmeier, C. Investigating the Relativistic Motion of the Stars Near the Supermassive Black Hole in the Galactic Center. Astrophys. J. 2017, 845, 22. [CrossRef]

75. Eckart, A.; Schödel, R.; Meyer, L.; Trippe, S.; Ott, T.; Genzel, R. Polarimetry of near-infrared flares from Sagittarius A*. Astron. Astrophys. 2006, 455, 1-10. [CrossRef]

76. Eckart, A.; Hüttemann, A.; Kiefer, C.; Britzen, S.; Zajaček, M.; Lämmerzahl, C.; Stöckler, M.; Valencia-S, M.; Karas, V.; García-Marín, M. The Milky Way's Supermassive Black Hole: How Good a Case Is It? Found. Phys. 2017, 47, 553-624. [CrossRef]

77. Landau, L.D.; Lifshitz, E.M. The Classical Theory of Fields; Pergamon Press: Oxford, UK, 1976.

78. Poisson, E. The Motion of Point Particles in Curved Spacetime. Living Rev. Relativ. 2004, 7, 6. [CrossRef]

79. DeWitt, B.S.; Brehme, R.W. Radiation damping in a gravitational field. Ann. Phys. 1960, 9, 220-259. [CrossRef]

80. Hobbs, J. A vierbein formalism of radiation damping. Ann. Phys. 1968, 47, 141-165. [CrossRef]

81. Chrzanowski, P.L.; Misner, C.W. Geodesic synchrotron radiation in the Kerr geometry by the method of asymptotically factorized Green's functions. Phys. Rev. D 1974, 10, 1701-1721. [CrossRef]

82. Gal'tsov, D.V. Radiation reaction in the Kerr gravitational field. J. Phys. Math. Gen. 1982, 15, 3737-3749. [CrossRef]

83. Sokolov, A.A.; Ternov, I.M.; Aliev, A.N.; Gal'tsov, D.V. Synchrotron radiation in curved space-time. Sov. Phys. J. 1983, 26, 36-40. [CrossRef]

84. Sokolov, A.A.; Gal'tsov, D.V.; Petukhov, V.I. Radiation emitted by relativistic particles moving in the vicinity of the Schwarzschild black hole, immersed in an external magnetic field. Phys. Lett. A 1978, 68, 1-2. [CrossRef]

85. Shoom, A.A. Synchrotron radiation from a weakly magnetized Schwarzschild black hole. Phys. Rev. D 2015, 92, 124066. [CrossRef]

86. Piotrovich, M.Y.; Silant'ev, N.A.; Gnedin, Y.N.; Natsvlishvili, T.M. Magnetic fields and quasi-periodic oscillations of black hole radiation. Astrophys. Bull. 2011, 66, 320-324. [CrossRef]

87. Baczko, A.K.; Schulz, R.; Kadler, M.; Ros, E.; Perucho, M.; Krichbaum, T.P.; Böck, M.; Bremer, M.; Grossberger, C.; Lindqvist, M.; et al. A highly magnetized twin-jet base pinpoints a supermassive black hole. Astron. Astrophys. 2016, 593, A47. [CrossRef]

88. Zajacek, M.; Tursunov, A. The Electric Charge of Black Holes: Is It Really Always Negligible. Obesity 2019, 139, $231-236$.

89. Tursunov, A.; Zajaček, M.; Eckart, A.; Kološ, M.; Britzen, S.; Stuchlík, Z.; Czerny, B.; Karas, V. Effect of Electromagnetic Interaction on Galactic Center Flare Components. Astrophys. J. 2020, 897, 99. [CrossRef]

90. Weingartner, J.C.; Draine, B.T.; Barr, D.K. Photoelectric Emission from Dust Grains Exposed to Extreme Ultraviolet and X-Ray Radiation. Astrophys. J. 2006, 645, 1188-1197. [CrossRef] 
91. Tursunov, A.; Bakhtinur, J.; Stuchlík, Z.; Kološ, M. Electric Penrose process: high-energy acceleration of ionized particles by non-rotating weakly charged black hole. Phys. Rev. D 2021, 104, 084099. [CrossRef]

92. Stuchlík, Z.; Charbulák, D.; Schee, J. Light escape cones in local reference frames of Kerr-de Sitter black hole spacetimes and related black hole shadows. Eur. Phys. J. C 2018, 78, 180. [CrossRef] 